\title{
1 An ancient route towards salicylic acid and its implications for the perpetual 2 Trichormus-Azolla symbiosis
}

4 Sophie de Vries ${ }^{1 *}$, Cornelia Herrfurth ${ }^{2,3}$, Fay-Wei Li $i^{4,5}$, Ivo Feussner ${ }^{2,3,6}$, Jan de Vries ${ }^{7,8,9}$

5 1- Heinrich-Heine University Düsseldorf, Population Genetics, Universitätsstr. 1, 40225 Düsseldorf, Germany

62 - University of Goettingen, Albrecht-von-Haller-Institute for Plant Sciences, Department of Plant Biochemistry, Justus-von-

7 Liebig Weg 11, 37077 Goettingen, Germany

83 - University of Goettingen, Goettingen Center for Molecular Biosciences (GZMB), Goettingen Metabolomics and Lipidomics

9 Laboratory, Justus-von-Liebig Weg 11, 37077 Goettingen, Germany

10 4-Boyce Thompson Institute, Ithaca, NY, USA.

115 - Plant Biology Section, Cornell University, Ithaca, NY, USA

12 6-University of Goettingen, Goettingen Center for Molecular Biosciences (GZMB), Department of Plant Biochemistry, Justus-

13 von-Liebig Weg 11, 37077 Goettingen, Germany

14 7-University of Goettingen, Institute for Microbiology and Genetics, Department of Applied Bioinformatics, Goldschmidtstr. 1, 1537077 Goettingen, Germany

168 -University of Goettingen, Goettingen Center for Molecular Biosciences (GZMB), Department of Applied Bioinformatics,

17 Goldschmidtsr. 1, 37077 Goettingen, Germany

18 9-University of Goettingen, Campus Institute Data Science (CIDAS), Goldschmidstr. 1, 37077 Goettingen, Germany

20 *Corresponding authors:

21 Sophie de Vries: rommels@hhu.de

22 Jan de Vries: devries.jan@uni-goettingen.de

\section{ABSTRACT}

Despite its small size, the water fern Azolla is a giant among plant symbioses. Within each of its leaflets, a specialized leaf cavity is home to a population of nitrogen-fixing cyanobacteria (cyanobionts). While examples of nitrogen fixing cyanobionts are found across the land plant tree of life, Azolla is unique in that its symbiosis is perpetual: the cyanobionts are inherited during sexual and vegetative propagation of the fern. What underpins the communication between the two partners? In angiosperms, the phytohormone salicylic acid (SA) is a wellknown regulator of plant-microbe interactions. Using HPLC-MS/MS, we pinpoint the presence of SA in the fern; using comparative genomics and phylogenetics, we mined homologs of SA biosynthesis genes across Chloroplastida (Viridiplantae). While canonical isochorismate synthase (ICS) sequences are largely limited to angiosperms, homologs for

35 the entire Phenylalanine ammonia-lyase (PAL)-dependent pathway likely existed in the last common ancestor of land plants. Indeed, A. filiculoides secondarily lost its ICS, but has the genetic competence to derive SA from benzoic acid. Global gene expression data from

38 cyanobiont-containing and -free $A$. filiculoides unveil a putative feedback loop: SA appears

39 to induce cyanobacterial proliferation, which in turn down-regulates genes in SA 40 biosynthesis and its responses. 


\section{INTRODUCTION}

44 The water fern Azolla filiculoides is best-known for its unique symbiosis with a nitrogen-fixing 45 cyanobacteria, hereafter referred to as the cyanobionts (Rai et al., 2000). Unlike any other 46 nitrogen-fixing symbiosis, the one of Azolla and its cyanobiont is vertically inherited from generation to generation (Peters and Meeks 1989, Zheng et al. 2009a, de Vries and de Vries 2018). Indeed, the cyanobiont cannot live without its host (Peters and Meeks 1989, Zheng et al. 2009b). This dependency is written in its genome, too (Ran et al. 2010): the genome has undergone a strong reduction and erosion. On the other side, when wellsupplied with nitrogen nutrients Azolla is-in the lab-capable of living without its cyanobionts (Shi and Hall 1988). Yet, in nature the two partners occur together.

The Azolla cyanobiont is a section IV (Rippka et al. 1979), filamentous nitrogen-fixing cyanobacterium; taxonomically it is most commonly assigned to the genus Anabaena or Nostoc-but this affiliation is still debated (Pereira \& Vasconcelos 2014; de Vries and de Vries 2018). As of late it is called Trichormus azollae (Pereira \& Vasconcelos 2014). The cyanobiont lives in the leaf cavities of Azolla species (Peters and Meeks 1989) and cooccurs with several other bacterial associates, some which are denitrifiers, likely benefitting from the cyanobiont (Dijkhuizen et al. 2018). Additionally, some of the co-occurring bacteria appear to be inherited together with the cyanobiont (Zheng et al. 2009b).

The life cycle of Azolla is intertwined with that of its cyanobiont (Rai et al. 2000; Zheng et al. 2009a; de Vries and de Vries, 2018). During vegetative propagation (i.e. simple growth of the fern body and break-up into clonal "colonies"), cyanobiont populations are transferred to new leaf cavities via specialized trichomes (Calvert et al. 1985; Hill 1989). The trichomes grow out of the newly forming leaf cavities and get associated to the shoot apical meristem 68 (Calvert and Peters 1981, Zheng et al. 2009b). On the apical meristem is a colony of 69 hormogonia-like filaments of the cyanobiont (Peters et al. 1978, Peters and Meeks 1989); these filaments become attached to the branched trichome and as the leaf grows, both the trichome and the cyanobiont filaments are engulfed by the formed leaf cavity (Peters and Meeks 1989, Zheng et al. 2009a). Whether the other bacteria are transferred by the same means and whether as a result each leaf cavity is equipped with a highly similar microbiome

74 is currently not known. During sexual propagation, the cyanobionts are attracted to the 75 developing sporocarps, where they, similar to the vegetative propagation, become entangled 76 in trichomes (Becking, 1987; Perkins and Peters 1993). While the formation of the indusium

77 cell layer around the spores occurs, the cyanobionts are moved upwards to the tips of the 
sporocarps, where they enter through the indusium pore into the forming indusium chamber (Perkins and Peters 1993). Upon maturation of the sporocarp, the cyanobionts in this chamber turn into resilient akinetes, facilitating the co-dormancy of symbiont and host (Perkins and Peters 1993, Zheng et al. 2009b). Hence, the process of sporocarp maturation and cyanobiont differentiation appears to be tightly coordinated (Zheng et al. 2009b). The molecular mechanisms that underpin this coordination are however unknown.

Vegetative propagation has been studied with regard to its chemical set-up. It appears that especially the trichomes are rich in phenolic compounds (Pereira \& Carrapiço 2007). And it has been hypothesized that these phenolics may be relevant in some way for the transfer of cyanobionts from one cavity to another (Pereira \& Carrapiço 2007). Indeed, it has been highlighted that the expression of a chalcone synthase (CHS) homolog of $A$. filiculoides is both responding to the absence of nitrogen and the absence of cyanobionts from the leaf pockets ( $\mathrm{Li}$ et al. 2018, Eily et al. 2019). A recent study identified homologous genes encoding enzymes of the flavonoid biosynthesis pathway and functionally characterized a key enzyme, leucoanthocyanidin reductase (LAR) (Güngör et al. 2021). The identified homologs of the flavonoid biosynthetic genes (homologs of chalcone isomerase $(\mathrm{CHI}), \mathrm{CHS}$ and dihydroflavonol 4-reductase (DFR) and $A f L A R)$ appear co-expressed during the diurnal cycle of $A$. filiculoides independent of the nitrogen supply (Güngör et al. 2021). However, without nitrogen the genes rapidly decrease in expression level during the night cycle (Güngör et al. 2021).

Among the flavonoids that have been identified in $A$. filiculoides and also another water fern, Azolla pinnata, are derivatives of Caffeoylquinic acid, Dicaffeoylquinic (Epi-)Catechin, Quercitin and Kaempferol (Güngör et al. 2021). These flavonoids are involved in defense responses in other plants, e.g. the gymnosperms Picea abies (Danielsson et al. 2011). Similarly, another phenolic compound involved in plant defense, salicylic acid (SA) (Ding and Ding 2020), has also been implicated in altering the abundance of the cyanobionts and its expression of genes involved in nitrogen fixation (de Vries et al. 2018). This may suggest that a link between this highly coordinated symbiosis and the defense capabilities of Azolla has evolved in the 66-100mya old association (Hall and Swanson 1968, Collinson 2002; Carrapiço 2006).

SA can be synthesized via two distinct routes in land plants: In Arabidopsis thaliana the majority of SA stems from conversion of chorismate into isochorismate by isochorismate synthase (ICS) (Wildermuth et al. 2001, Garcion et al. 2008). Isochorismate is transferred from the chloroplast to the cytoplasm and as a substrate of avrPphB SUSCEPTIBLE3 
115 (PBS3) conjugated with glutamate, resulting in the product isochorismate-9-glutamate 116 (Rekhter et al. 2019). A spontaneous reaction, which can be also catalysed by ENHANCED 117 PSEUDOMONAS SUSCEPTIBILTY 1 (EPS1), results in the conversion of isochorismate-9118 glutamate to SA (Rekhter et al. 2019, Torrens-Spence et al. 2019). Yet, EPS1 appears to be 119 specific to Brassicaceae and a clear PBS3 ortholog has not been identified in $A$. filiculoides 120 (de Vries et al. 2018, Torrens-Spence et al. 2019, Li et al. 2020).

121

122 Already in other angiosperms than A. thaliana, SA may not come from the ICS-dependent 123 pathway but rather derives from benzoic acid (Meulwy et al. 1995, Pallas et al. 1996, 124 Coquoz et al. 1998). These early publications suggested that SA is synthesized via a 125 Phenylaline Ammonia Lyase (PAL)-dependent pathway from phenylalanine. Phenylalanine 126 is converted into trans-cinnamic acid (Widhalm and Dudareva 2015). From here, SA can be 127 synthesized via peroxisomal $\beta$-oxidation or two cytoplasm-localized non-oxidative pathways, 128 one CoA-dependent and one CoA-independent (Widhalm and Dudareva 2015). None of the 129 pathways has so far full enzymatic evidence. Yet, some steps along the way have been 130 functionally characterized. Some first importers of phenylpropanoid pathway-derived 131 compounds into the peroxisome have been identified (Block et al., 2014). In the oxidative 132 peroxisomal pathway, trans-cinnamic acid is converted into cinnamoyl-CoA (if the latter is 133 not imported) via trans-cinnamic acid ligase ( $P h-\mathrm{CNL})$ from Petunia hybrida and possibly 134 also by its ortholog BENZOYLOXYGLUCOSINOLATE 1 (BZO1) in A. thaliana (Colquhoun et 135 al. 2012, Klempien et al. 2012, Lee et al. 2012). In P. hybrida the following steps towards 136 benzoyl-CoA are catalyzed via chalcone dehydrogenase (PhCHD) and katalase1 (PhKAT1) 137 (Van Moerkercke et al. 2009, Qualley et al. 2012). In A. thaliana they are suggested to be 138 realized by the multifunctional $\beta$-oxidation enzyme abnormal inflorescence meristem1 139 (AtAIM1) and AtKAT2 (Bussell et al. 2014, Widhalm and Dudareva 2015). The resulting 140 benzoyl-CoA is converted to benzoic acid by a thioesterase, likely 1,4-dihydroxy-2-naphthoyl 141 (DHNA)-CoA THIOESTERASE 1 (AtDHNAT1/2) in A. thaliana (Widhalm et al. 2012, 142 Widhalm and Dudareva 2015) or Ph-TE1 in P. hybrida (Adebesin et al. 2018). In the non143 oxidative cytoplasmic pathways, a hydratase is suggested to use trans-cinnamic acid or 144 cinnamoyl-CoA as a substrate and hydrates the double bond to form 3-hydroxy-3145 phenylpropionic acid or 3-hydroxy-3-phenylpropanoyl-CoA, respectively (see Widhalm and 146 Dudareva, 2015). Both of which are likely converted into benzaldehyde by a lyase147 dependent reaction (see Widhalm and Dudareva, 2015). In $A$. thaliana benzaldehyde is 148 converted to benzoic acid by Arabidopsis Aldehyde Oxidase4 (AAO4) (Ibdah et al. 2009), 149 while in Snapdragon (Antirrhinum majus) it is imported into the mitochondrion and converted 150 to benzoic acid by benzaldehyde dehydrogenase (BALDH) (Long et al. 2009). Independent 
151 of the route benzoic acid is produced, it was suggested that benzoic acid 2-hydroxylase

152 (BA2H)-like enzyme catalyzes the last step from there to SA (León et al. 1995).

153

154 In this study we explore the connections between SA and the coordinated symbiosis of the 155 water fern $A$. filiculoides and its cyanobiont $T$. azollae. Using HPLC-MS/MS we measured $156 \mathrm{SA}$ in roots and the photosynthesizing sporophyte body of $A$. filiculoides. Because it is not 157 known by which pathway the symbiotic system synthesizes SA, we used the cumulated 158 knowledge on enzymatic synthesis of SA of the green lineage to identify candidate genes for 159 SA biosynthesis in A. filiculoides. Applying comparative genomics and phylogenetics, we 160 searched for orthologs_and the closest homologs_of key genes likely acting in the ICS161 and PAL-dependent pathway; we further explored the possibility of cyanobiont-derived SA. 162 We find that only the genomes of Chara braunii, Selaginella moellendorffii and angiosperms encode a separate standalone ICS. All other chlorophyte and streptophyte algae as well as bryophytes and the fern Salvinia cucullata contain a fusion enzyme, where the ICS domain is fused with a MenC and/or MenD domain, suggesting that chorismate can be quickly diverted into the phylloquinone pathway. Moreover, A. filiculoides entirely lacks an ICSencoding gene. In contrast, our phylogenetic data support the presence of homologs for all known steps in the PAL-dependent pathway across land plants, suggesting that the benzoic acid pathway has the potential to be recruited for SA biosynthesis in several land plant lineages. Our data pinpoint the biosynthetic routes of SA via benzoic acid as the most likely origin of SA in A. filiculoides. We carried out global gene expression analyses on the PALdependent SA biosynthesis pathway and homologs to SA responsive genes. The majority of SA-associated genes that showed differential gene expression patterns appear to be downregulated in the presence of the cyanobionts. Integrating this with previous results, we hypothesize that a feedback loop has co-evolved between the two partners, that tightly coordinates the levels of SA with the proliferation of the cyanobionts.

\section{MATERIAL AND METHODS}

\section{Measurement of SA levels}

The water fern Azolla filiculoides was cultured at $75 \%$ relative humidity in a heat-sterilized glass vessel. The ferns floated on $250 \mathrm{~mL}$ filtered water with a $\mathrm{pH}$ of 7.0 . During the $16 \mathrm{~h}-$ long day, the fern was exposed to $450 \mu \mathrm{mol}$ quanta $\mathrm{m}^{-2} \mathrm{~s}^{-1}$ at $24{ }^{\circ} \mathrm{C}$; during the $8 \mathrm{~h}$-long night, the temperature dropped to $20^{\circ} \mathrm{C}$. We picked plants from actively growing culture and used sterilized micro-scissors to separate the fern body into whole roots and green sporophyte

186 tissue (i.e. with roots removed). Extraction was performed as previously described for lipids 187 (Matyash et al., 2008), with some modifications specified previously (Iven et al., 2012). The 
188 analysis was performed using an Agilent 1100 HPLC system (Agilent, Waldbronn, Germany)

189 coupled to an Applied Biosystems 3200 hybrid triple quadrupole/linear ion trap mass

190 spectrometer (MDS Sciex, Ontario, Canada) by using an ESI chip ion source (TriVersa

191 NanoMate; Advion BioSciences, Ithaca, NY, USA). The quantification of SA was performed

192 as previously described (Iven et al., 2012) applying a scheduled multiple reaction monitoring

193 detection in negative ionisation mode including the following MS transitions: 141/97

194 [declustering potential (DP) $-45 \mathrm{~V}$, entrance potential (EP) $-7 \mathrm{~V}$, collision energy (CE) -22 V]

195 for $\mathrm{D}_{4}-\mathrm{SA}$ and $137 / 93$ (DP -45 V, EP -7 V, CE -22 V) for SA. The quantification was carried

196 out using a calibration curve of intensity $(\mathrm{m} / z)$ ratios of $[S A] /\left[D_{4}-S A\right]$ vs. molar amounts of

197 SA (0.3-1000 pmol).

Statistical analyses have been performed in R v.3.6.0. Data was tested for normality using a

Shapiro-Wilks test (Shapiro and Wilk, 1965), following a test to compare the variance of the

201 data between replicates of SA measurements from roots and sporophytes of $A$. filiculoides.

202 The data was normally distributed and showed no significant difference in variance. Accordingly, a two-sample t-test (Student 1908) was computed.

For phylogenetic analyses, we worked with protein data from the genomes of Anthoceros

208 agrestis as well as Anthoceros punctatus (Li et al., 2020), Amborella trichopoda (Amborella 209 Genome Project, 2013), Arabidopsis thaliana (Lamesch et al., 2011), Azolla filiculoides (Li et 210 al., 2018), Brachypodium distachyon (The International Brachypodium Initiative, 2010), 211 Capsella grandiflora (Slotte et al., 2013), Gnetum montanum (Wan et al., 2018), Marchantia 212 polymorpha (Bowman et al., 2017), Nicotiana tabacum (Sierro et al., 2014), Oryza sativa 213 (Ouyang et al., 2007), Picea abies (Nystedt et al., 2013), Physcomitrium patens (Lang et al., 214 2018), Salvinia cucullata (Li et al., 2018), Selaginella moellendorffii (Banks et al., 2011), and 215 Theobroma cacao (Argout et al., 2011); (b) the genomes of seven streptophyte algae: 216 Chlorokybus atmophyticus (Wang et al., 2020), Chara braunii (Nishiyama et al., 2018), 217 Klebsormidium nitens (Hori et al., 2014), Mesotaenium endlicherianum (Cheng et al., 2019), 218 Mesostigma viride (Wang et al., 2020), Penium margaritaceum (Jiao et al., 2020), 219 Spirogloea muscicola (Cheng et al., 2019). For each of the protein families, specific cutoffs 220 were chosen (see below). All sequences that have met the respective cutoffs, were aligned 221 using MAFFT v7.453 (Katoh and Standley, 2013) with the setting L-INS-I. Alignments were 222 cropped to conserved and alignable regions for all homologs. Maximum likelihood 223 phylogenies were computed using IQ-TREE multicore v.1.5.5 (Nguyen et al., 2015) with 100 
224 bootstrap replicates. For finding the best model of protein evolution, we used ModelFinder 225 (Kalyaanamoorthy et al., 2017). The best models were LG+G4 (Le and Gascuel, 2008) for 226 PAL, LG+I+G4 for AIM1, KAT, LG+F+l+G4 for AAO, JTT+l+G4 (Jones et al., 1992) for 227 DHNAT.

Protein sequences for enzymes involved in PAL-dependent biosynthesis of SA in $A$. thaliana, P. hybrida or Snapdragon (Antirrhinum majus) have been downloaded from TAIR (Lamesch et al., 2011), NCBI, and UniProt. Sequences have sampled by using an HMM search via HMMER 3.1b2 (Mistry et al., 2013) with the ammonia lyase domain (obtained from PFAM) as a query against the proteins from the above-described genomes; all sequences that met the inclusion threshold were retained. Additionally, HAL and fungal PAL sequences that were included in de Vries et al. (2017) were added. For computing the maximum likelihood phylogeny, we used only those sequences that were at least 600 amino acids in length.

For identification of putative ICS sequences in A. filiculoides, we conducted an HMM search using HMMER 3.1b2 (Mistry et al., 2013) for the chorismate binding domain against the high- and low-confidence protein sequences from the A. filiculoides genome v.1.1 (Li et al., 2018). We used all three thusly identified Azolla sequences that met the HMM search inclusion threshold ( $e$ values of $4.8^{*} 10^{-87}, 4.6^{*} 10^{-85}$, and $3.7^{\star} 10^{-84}$ ) as queries for a BLASTp search against the protein dataset from the above described genomes. To improve the sampling on the closest algal relatives of land plants, we additionally included sequences found via a tBLASTn in the transcriptomes of Spirogyra pratensis (de Vries et al., 2020), Zygnema circumcarinatum (de Vries et al., 2018), and Coleochaete orbicularis (Ju et al., 2015). We used only those sequences that have met an e value cutoff of $10^{-10}$, had a minimum length of 300 amino acids.

\section{Identification of SA biosynthesis genes in cyanobacteria}

252 To identify the ICS of $T$. azollae we downloaded the ICS sequence of Trichormus variabilis

253 from NCBI and used it in a BLASTp query against $T$. azollae in the non-redundant protein

254 (nr) database of NCBI. To identify chorismate binding enzymes we blasted ICS, anthranilate

255 synthase and aminodeoxychorismate synthase of $T$. azollae against cyanobacteria in the $\mathrm{nr}$ 256 database. Further, we downloaded sequences for isochorismate pyruvate-lyase (IPL) from 257 Pseudomonas aeruginosa (accession NP_252920.1) and salicylate synthase (SAS) of 258 Mycobacterium tuberculosis (accession YP_177877.1). We used these sequences to 259 identify IPL and SAS enzymes in cyanobacteria using BLASTp at NCBI. 


\section{Identification of homologs to SA responsive genes}

262 We downloaded all protein sequences from TAIR10 (Lamesch et al. 2011) linked to the term

263 response to SA. This resulted in 176 sequences, which we used as a query in a blastp

264 search against the proteome of $A$. filiculoides ( $\mathrm{Li}$ et al. 2018). The 16,776 non-unique hits

265 were funneled into a reciprocal BLASTp search with max_target_seqs 1, returning the best

266 hits from $A$. thaliana for 3,386 unique $A$. filiculoides proteins. We next retained only those

267 sequences that had their best hit to one of the 176 accessions of $A$. thaliana associated with

268 the term response to SA and used an e-value cutoff of $10^{-7}$. The $A$. filiculoides sequences

269 corresponding to the final list of hits against $A$. thaliana were considered homologs to SA

270 responsive genes; and after removal of low-confidence proteins added up to 97 candidates.

271

\section{Analyses of conserved sites in chorismate binding enzymes}

273 Protein sequences of the chorismate binding enzymes of plants were separated into ICS,

274 anthranilate synthase and aminodeoxychorismate synthase sequences according to the

275 phylogenetic analysis. Further protein sequences of the top 100 blastp hits for each type of

276 the cyanobacterial chorismate binding enzymes were downloaded from NCBI. Each subset

277 of protein sequences was aligned with that of the anthranilate synthase of Salmonella

278 enterica subsp. enterica serovar Typhimurium str. LT2 with MAFFT using a L-INS-I

279 approach (Katoh and Standley, 2013) to identify the conserved positions described in Plach

280 et al. (2015). To generate sequence logos for the respective groups the sequence from

281 Salmonella was removed from the alignment after the positions were located. Sequence

282 logos were generated based on the alignments.

283

\section{Transcriptomic profiling of candidate genes for SA biosynthesis and signaling}

285 Raw read count data for $A$. filiculoides with or without cyanobiont and treated with or without 286 fixed nitrogen $\left(\mathrm{NH}_{4} \mathrm{NO}_{3}\right)$ supply were obtained from Eily et al. (2019). We calculated the 287 TPM for all datasets according to (Robinson and Oshlack, 2010) and used edgeR (Robinson 288 et al., 2010) to calculate $\log _{2}$-foldchange and to identify the differently expressed genes 289 (DEGs) within a given dataset. To calculate FDR from p-values a Benjamini-Hochberg 290 correction was used.

291

\section{Protein domain search}

293 We used Interpro (Blum et al., 2020) to identify protein domains in chorismate binding 294 enzymes of land plants and used the information of TIGR to identify the putative function of 295 the chorismate binding enzymes identified in our BLASTp and phylogenetic analyses. 296 Likewise, we used CD search (Marchler-Bauer et al., 2004) and the information of TIGR IDs 
297 for protein domain analyses of cyanobacterial protein domains of chorismate binding 298 enzymes.

\section{RESULTS AND DISCUSSION}

301

\section{Azolla filiculoides produces SA}

303 Molecular data on cross-talk and co-ordination between Azolla and its cyanobionts are just 304 becoming more and more abundant (Brouwer et al. 2017, de Vries et al. 2018, Li et al. 2018,

305 Eily et al. 2019, Güngör et al. 2020). Several studies point to a role of flavonoids in the 306 communication between the two partners (Pereira and Carrapiço 2007, Güngör et al. 2020)

307 and MeSA, a methylated, volatile derivative of SA, appears to also interfere in the interaction 308 (de Vries et al. 2018). Yet, we do not know whether and how SA is synthesized 309 endogenously in $A$. filiculoides. In a first step, we measured endogenous SA levels in the roots and sporophyte of $A$. 312 filiculoides (Table 1). The data confirmed that $A$. filiculoides harbors SA. Roots appear to 313 harbor slightly more SA than the sporophyte (roots: $0.18 \pm 0.03 \mathrm{nmol} / \mathrm{g}$ fresh weight (FW), 314 sporophyte: $0.09 \pm 0.02 \mathrm{nmol} / \mathrm{g} \mathrm{FW}, \mathrm{p}$-value=0.0337). Yet, these basal levels of $\mathrm{SA}$ in the 315 non-axenic Azolla are relatively low compared to for example Col-0 from $A$. thaliana 316 (Rekhter et al. 2019). Regardless of how much SA was measured, the mere fact that it is 317 present in the fern body begs the question of how it is synthesized. We thus next used the 318 recently released genomic data on Azolla filiculoides to explore possible biosynthetic routes towards SA.

\section{A treatise of the three possible routes for SA biosynthesis in A. filiculoides}

322 Land plants have two biosynthetic routes towards SA. One route depends on ICS, the other 323 on PAL (Vlot et al. 2009). Unlike other land plants, Azolla theoretically has a third option to 324 acquire SA via the biochemical capacities of its omnipresent cyanobiont. Here we used a 325 phylogenetic approach utilizing the genome data of $A$. filiculoides and a diversity of genomes 326 from other species from the green lineage to identify putative candidates for the SA 327 biosynthesis pathways in Azolla. We further investigated the cyanobiont genome with regard 328 to its theoretical ability to synthesize the hormone.

331 The ICS-pathway is localized in the plastid and cytoplasm and requires ICS1 and 2 and 332 PBS3 (Wildermuth et al. 2001, Garcion et al. 2008, Rekhter et al. 2019). The last step in the 
333 conversion to SA can either be spontaneous or catalyzed by EPS1 (Rekhter et al. 2019,

334 Torrens-Spence et al. 2019). EPS1 is Brassicaceae-specific (Torrens-Spence et al. 2019),

335 but it is also not strictly required for the interaction. PBS3 has emerged from a lineage-

336 specific expansion of GH3-encoding genes (de Vries et al. 2018, Li et al. 2020). However,

337 two GH3-homologs exist in the A. filiculoides genome that fall into the same GH3 clade as

338 AtPBS3 ( $\mathrm{Li}$ et al. 2018, Li et al. 2020 supplemental material). For the key enzyme, ICS,

339 BLASTp surveys identified only a single low-confidence sequence in A. filiculoides (Li et al.

340 2018, 2020), which appears rather divergent to other ICS1 candidates as shown in the

341 (supplemental) phylogenetic analyses included in Li et al. (2020). Here, we investigated the

342 chorismate binding protein families in more detail.

344 Using an HMMsearch against the genome of $A$. filiculioides, we found three protein-coding 345 genes (Azfi_s0185.g056617, Azfi_s0002.g007267, and Azfi_s0061.g034905) with a 346 chorismate-binding domain (for which we obtained an HMM profile from PFAM; El-Gebali et 347 al., 2019). ICS sequences exist throughout the green lineage, but we found no ortholog for 348 A. filiculoides (Figure 1a). The existence of an ICS sequence in land plants and algae 349 suggests that the last common ancestor of the Chloroplastida had an ICS sequence. The 350 lack of an ICS in A. filiculoides can only be explained by a secondary loss; this absence is 351 corroborated by the lack of clear ICS orthologs in the transcriptome data of Azolla carolinia 352 (sequenced in the framework of the 1KP efforts; Carpenter et al., 2019) and Azolla pinnata 353 (Shen et al., 2018; see supplemental Figure S1).

355 Whether the isochorismate pathway is used in all species with an ICS ortholog is however 356 unclear when we investigated the protein domain structures of all ICS sequences. The ICS 357 candidates from bryophytes, algae and the fern S. cucullata appear to be compound 358 enzymes coupled with a MenC and/or MenD domain (Figure 1a). This suggests that in these 359 species ICS may directly funnel isochorismate into the menaquinone pathway rather than 360 into a route towards SA. Yet, in some of the species, such as the moss Physcomitrium 361 patens, SA has been detected (Ponce De León et al., 2012). Thus, ICS was either recruited 362 later in the evolution of land plants as the primary route towards SA or it is species specific 363 whether SA derives from isochorismate or benzoic acid in plants. However, ICS may not be 364 the only source for isochorismate.

366 Usually anthranilate synthases convert chorismate to aminodeoxyisochorismate and then to 367 anthranilate. That said, a Salmonella anthranilate synthase was successfully engineered to 368 become an isochorismate-forming enzyme by exchanging two amino acids in the active 369 center (Plach et al. 2015). A mutation leading to a lysine at position 263 instead of glutamine 
370

371

372

373

374

375

376

377

378

379

380

381

382

383

384

385

386

387

388

389

390

391

392

393

394

395

396

397

398

399

400

401

402

403

404

405

406

and another either at 364 changing a methionine to a lysine or at 365 changing lysine to valine led to isochorismate formation in Salmonella (Plach et al. 2015). These residues are more or less conserved across different phyla in bacteria (Plach et al. 2015): with Q263 and M364 being conserved across all analyzed anthranilate and aminodeoxychorismate synthases and L365 in the anthranilate synthases and 1365 in the aminodeoxychorismate synthases. ICS and salicylate synthase (SAS) show a conserved K263, but appeared to be slightly more variable at positions 364 (ICS major amino acid (aa): 364L; SAS major aa: 364I) and position 365 (ICS major aa: 365V; SAS major aa: 365S). Further, the 263 lysine (K190 in Escherichia coli) was shown to be highly relevant for the function of bacterial ICS (Kolappan et al. 2007). We found that all land plant lineages included in this phylogeny, including $A$. filiculoides, also encode putative anthranilate synthases in their genomes (Figure 1a).

To elucidate whether the putative anthranilate synthases of Chloroplastida and of $A$. filiucloides in particular can potentially take over the role of ICS in the fern, we explored the respective positions determining the product specificity (a) in the set of land plant chorismate binding enzymes to establish the most abundant residues in land plants at these functionally relevant positions and (b) in the three $A$. filiculoides protein sequences. The anthranilate synthases of plants had the same aa pattern as bacterial anthranilate synthases: $Q, M$, and $L$ (Figure 1b), while the aminodeoxychorismate synthases encoded mainly an $E$ at the position equivalent to 263 , and $\mathrm{M}$, and I at the respective positions for 364 and 365 (Figure 1b). 263E was the second most abundant residue in aminodeoxychorismate synthases of bacteria (Plach et al. 2015), suggesting that the three residues are in general conserved across prokaryotes and eukaryotes. Plant ICS however showed a K, I and V at the respective positions in the alignment. All three positions were not variable in plant ICS (Figure 1b). This is corroborated by the fact, that the anthranilate synthase from $A$. filiculoides encoded a QML motif as any other land plant. Overall, this suggests that it does not fill in for the function of the missing ICS.

\section{The PAL-dependent pathway}

A PAL-dependent pathway for SA biosynthesis has been suggested for several angiosperms (Meuwly et al. 1995, Pallas et al. 1996, Coquoz et al. 1998). The synthesis of SA via PAL can be facilitated via several different routes of benzoic acid metabolism. All of them have in common that PAL, which converts phenylalanine to trans-cinnamic acid, is the entry point for SA synthesis (Widhalm and Dudareva, 2015). The $\beta$-oxidation pathway requires enzymes from the $4 \mathrm{CL}$ family and the closely related $A t B Z O 1 / P h-C N L$ clade, the hydratase PhCHD, PhKAT1and AtDHNAT1/2 (Van Moerkercke et al., 2009, Colquhoun et al., 2012, Klempien 
407 et al., 2012, Lee et al., 2012, Qualley et al., 2012, Widhalm et al., 2012, Widhalm and 408 Dudareva, 2015). The non-oxidative pathway also requires 4CL, the hydratase AIM1, which 409 is the ortholog of $A$. thaliana to PhCHD, a lyase, AAO4 (lbdah et al., 2009, Bussel et al., 410 2014, Widhalm and Dudareva, 2015) or in Snapdragon (Antirrhinum majus) the 411 benzaldehyde dehydrogenase (BALDH; Long et al. 2009). The last step in the biosynthesis 412 of SA is only indirectly characterized and an inhibitor study suggests that BA2H-like enzyme 413 is required (León et al., 1995).

To identify PAL candidates in A. filiculoides, we performed an HMMsearch with the aromatic lyase motif (HMM profile obtained from PFAM; El-Gebali et al., 2019) against the genome of A. filiculoides as well as other representative species from across the streptophyte tree of life. The aromatic lyase motif is present in PAL, (P)TAL, TAM and PAL-tRNA synthase fusion proteins of plants. We used this dataset together with a subset of fungal PAL and eukaryotic HAL sequences (selected from the dataset of de Vries et al. 2017) to build a phylogeny.

In our phylogeny the fungal PAL, tRNA-PAL fusion proteins and eukaryotic HAL proteins each form their own clade (Figure 2). Yet, the functionally characterized PTAL and TAM sequences of plants fall into the midst of the putative PAL clade (Figure 2). A detailed study in Sorghum bicolor has identified two positions relevant for distinguishing PAL and PTAL (Jun et al. 2018): the presence of Phe at position 123 (relative to the characterized SbPTAL Sb04g026510) and Lys at position 443, were present in sequences with PAL activity, while SbPTALs showed His and Lys at the two respective positions. In OsTAM (Yan et al. 2015) these two positions are occupied by a Tyr and Asn respectively; the same combination of residues occurs in a $S$. bicolor PAL/PTAL candidate (Figure 2) that had neither tyrosine nor phenylalanine activity (Jun et al. 2018). To make more sense of the phylogenetic data, we mapped this sequence information of the two positions to the phylogeny.

While all HAL sequences occupied a $123 \mathrm{~S}$ and 443M, the tRNA synthesis fusion proteins showed a deletion of both residues. In contrast, most sequences that fall into the plant and fungal PAL clade occupy Lys at position 443 (relative to Sb04g026510), with the exception of one very divergent sequence from $M$. polymorpha (Mapoly0142s0036.1.p; 443H) and the subclade including OsTAM (LOC_Os11g48110.1), which showed Asn at position 443

440 (Figure 2). Position 123 was more variant among fungal and plant PALs: Most fungal PALs 441 have His at the relevant site, suggesting they may be PTALs instead. In contrast, most plant 442 PAL candidates have a Phe at the site of interest, with the exception of the subclades 443 including the monocot PTALs and TAM candidates and one $A$. thaliana PAL sequence 
444 (123Y in AtPAL3; Figure 2). In agreement, AtPAL3 was found to have the lowest catalytic 445 activity as phenylalanine ammonia lyase of all four PALs of $A$. thaliana (Cochrane et al. 446 2004). All seven included sequences of $A$. filiculoides, that were full-length and high447 confidence sequences, showed a $123 \mathrm{~F}$ and 443K (Figure 2), suggesting them as likely PAL 448 candidates.

450 Next, we investigated the acyl-activating enzyme (AAE) family that includes Ph-CNL and its 451 co-ortholog AtBZO1 (Figure S2), and all At4CLs. Our analysis captured sequences from five 452 of the seven predicted subfamilies of AAEs (Shockey et al., 2003). In general, we recovered 453 four of the AAE subfamilies with a bootstrap support of at least 75 . The family shows various 454 lineage-specific expansions (Figure S2). Only AAE clade VII did not form a monophyletic 455 clade (Figure S2). Clade I, IV, V and VI appear to have their origin in the last common 456 ancestor of land plants, yet especially clade IV to VI show substantial lineage-specific 457 radiation. Our enzymes of interest belong to these highly expanded clades: AtBZO1 and Ph$458 \mathrm{CNL}$ belong to AAE clade $\mathrm{VI}$, and $4 \mathrm{CL} 1,2,3$ and 5 belong to AAE clade IV (Figure S2, 459 Shockey et al., 2003). Despite that, we found four clear homologs of $A$. filiculoides for the 460 4CL-encoding genes (Azfi_s0003.g007625, Azfi_s0013.g013344, Azfi_s0114.g046013 and 461 Azfi_s0030.g024259) and two candidates for AAE clade VI (BZO1/Ph-CNL; 462 Azfi_s0159.g053935 and Azfi_s0002.g003721 [low-confidence sequence]). The next steps 463 in the oxidative and non-oxidative pathways are catalyzed by hydratases $P h \mathrm{CHD}$ in $P$. 464 hybrida and likely by its ortholog AtAIM1 in A. thaliana (Qualley et al. 2012, Bussell et al. 465 2014). Our phylogeny shows that an AIM1/CHD hydratase was present in the last common 466 ancestor of Chloroplastida. While several lineages have species-specific expansions of the 467 AIM1/CHD family, A. filiculoides possesses one clear ortholog for this hydratase 468 (Azfi_s0256.g060521; Figure 3). A similar picture emerges for the next step in the 469 peroxisomal pathway catalyzed by KAT: One KAT homolog was present in the last common 470 ancestor of Chloroplastida, but KAT sequences have expanded in several lineages, most 471 pronounced in the Brassicaceae (Figure 4). In contrast, the two sequenced fern genomes 472 (S. cucullata and $A$. filiculoides), however, possess only one copy for KAT 473 (Azfi_s0001.g000824, Figure 4). The final peroxisomal step to benzoic acid is realized by a 474 thioesterase; AtDHNAT1/2 shows activity on benzoyl-CoA esters (Widhalm et al. 2012) and 475 was hypothesized for this step (Widhalm and Dudareva 2015). The DHNAT family more or 476 less mirrors the species phylogeny, yet includes again species-specific radiations (Figure 5). 477 In the ferns, we find a duplication for DHNAT homologs in S. cucullata, but one ortholog in $A$. 478 filiculoides (Azfi_s0008.g011631; Figure 5). 
480 In the non-oxidative pathway, two possible routes towards benzoic acid exist. Both start in

481 the cytoplasm and require a hydratase (which is suggested to occur in both the cytosol and

482 the peroxisomal pathway; and a lyase to synthesize benzaldehyde (Widhalm and Dudareva,

483 2015). The conversion from benzaldehyde to benzoic acid can occur via two different steps:

484 (i) a cytoplasmic step, catalyzed by AAO4 in $A$. thaliana (Ibdah et al. 2009), or (ii) a 485 mitochondria-localized step, as described for Snapdragon (Antirrhinum majus), where

486 BALDH is converting the benzaldehyde to benzoic acid (Long et al. 2009). Given that for the

487 last step towards benzoic acid different routes processed by distinct types of enzymes have

488 been described, we analyzed both of them bioinformatically. Similar to the peroxisomal

489 pathway, BALDH and AAO homologs must have already existed in the common ancestor of

490 all Chloroplastida (Figure 6, S2). While algae contain only one homolog of BALDH, many of

491 the land plant lineages show lineage-specific expansions on BALDH-like homologs (Figure

492 S3). This also includes the fern $A$. filiculoides. In the ALDH2 clade that contains BALDH the

493 fern S. cucullata has an ortholog, but not $A$. filucloides (Figure S3). That said, several

494 sequences of $A$. filiculoides fall into the larger clade of ALDH1 and ALDH2 sequences,

495 where they, however, only cluster with weak support (bootstrap value 61) with the ALDH1

496 rather than ALDH2 sequences. These are therefore the most likely candidates for a BALDH

497 reaction (Azfi_s0021.g015740 [low-confidence sequence], Azfi_s0078.g038198,

498 Azfi_s0049.g030778 and Azfi_s0083.g038897; Figure S3). As for the BALDH-like

499 sequences, the AAO family shows lineage-specific radiation. This is most extensive in

500 angiosperms and the lycophyte $S$. moellendorffii (Figure 6). In contrast, the two ferns have

501 each only one copy for AAO (for A. filiculoides: Azfi_s0158.g053886; Figure 6).

502

503 The PAL-dependent pathway is less-explored as a source for SA in land plants compared to

504 ICS-dependent synthesis of SA. The PAL-dependent pathway, despite being highly radiated,

505 has homologs for all enzymes being present at least in the last common ancestor of all

506 streptophytes - going hand in hand with the idea that parts of this pathway were part of the

507 genetic building blocks that allowed for the radiation of spezialized metabolism in land plants

508 (Fürst-Jansen et al., 2020). This is most likely explained by the fact that this pathway is not a

509 highly specialized pathway for SA biosynthesis, but instead recruits steps from other

510 pathways, such as phenylpropanoid. With regard to the water fern $A$. filiculoides, our data

511 adds more support to benzoic acid-derived SA than isochorismate-derived SA biosynthesis

512 (Figure 7). In this unique system, however, another plausible route for synthesizing SA

513 exists. Cyanobacteria from the Nostocaceae, to which the cyanobiont belongs, can

514 synthesize SA (Toribio et al. 2020) and it is possible that $A$. filiculoides has an alternative

515 reservoir for this phytohormone. 


\section{The cyanobionts' pathway}

519 Instead of producing SA autonomously, it is theoretically conceivable that the cyanobiont

520 produces SA for $A$. filiculoides. We therefore investigated the cyanobionts genome for its

521 genetic capability to synthesize SA. Bacteria produce SA either via ICS and IPL enzymes or

522 directly via salicylate synthase (Ankenbauer and Cox, 1988, Gaille et al., 2002, Zwahlen et

523 al., 2007). Indeed, in several Nostocaceae SA has been measured (Toribio et al. 2020). This

524 leaves the possibility that between Azolla and its cyanobiont a tight regulation of SA-derived

525 host responses has co-evolved.

526

527 We searched for ICS sequences in T. azollae using the ICS sequence from T. variabilis; we 528 retrieved three sequences annotated as ICS (WP_013190659.1; query coverage 97\%, \% 529 identity 68.63\%, e-value 0), anthranilate synthase component I (WP_013192196.1; query 530 coverage $63 \%$, \% identity $27.48 \%$, e-value $2^{*} 10^{-23}$ ) and aminodeoxychorismate synthase 531 component I (WP_013189914.1; query coverage $71 \%$, \% identity $27.54 \%$, e-value $1^{*} 10^{-28}$; 532 Figure 8a). Isochorismate is converted to salicylic acid via IPL (Gaille et al., 2002). 533 Therefore, we next searched for an IPL candidate in T. azollae. Using the functionally 534 characterized IPL of Pseudomonas aeruginosa (Gaille et al., 2002) as a query for a 535 BLASTp, we found no hits in the genome of $T$. azollae but in several other cyanobacteria, 536 including the order Nostocales and the family Nostocaceae, to which T. azollae belongs 537 (Figure 8b). Thus, while some Nostoc species are able to synthesize SA (Toribio et al. 538 2020), only three different species from the Nostocaceae encoded an IPL sequence (Figure $5398 b)$.

541 Some bacteria use salicylate synthase, which directly converts chorismate to SA. Therefore, 542 we queried the functionally characterized salicylate synthase from Mycobacterium 543 tuberculosis (Zwahlen et al. 2007) in a BLASTp search against (a) T. azollae (supplemental 544 data S1A) and (b) all cyanobacteria (supplemental data S1B). In the search against $T$. 545 azollae we retrieved two hits; one against anthranilate synthase component I (e-value 6*10

$546^{28}$ ), of which the best hit in a protein domain search was indeed against anthranilate 547 synthase component I (TIGR00564, e-value 0) and one against ICS (e-value $2^{*} 10^{-18}$ ), but no 548 hit to an annotated salicylate synthase. In contrast to the IPL distribution, the most hits of 549 salicylate synthase were in the family of Nostocaceae (Figure 8c). All of the top100 salicylate 550 synthase-like sequence from diverse cyanobacteria, were predicted to contain a salicylate 551 synthase domain (TIGR03494). Yet, all sequences also matched TIGR domain annotations 552 for an ICS domain (TIGR00543), an aminodeoxychorismate synthase component I domain 553 (TIGR00553) and an anthranilate synthase component I domain (TIGR00564). The positions 
554 for all domain annotations were partially overlapping. This is in contrast to the two

555 sequences retrieved for $T$. azollae, which had their best TIGR domain hits to an anthranilate

556 synthase component I (TIGR00564, e-value 0) and ICS domain (TIGR00543, e-value

$\left.5574.75^{*} 10^{-107}\right)$.

558

559 As described above engineered anthranilate synthases from Salmonella had been described

560 to convert chorismate to isochorismate instead of anthranilate (Plach et al. 2015). Some of

561 these anthranilate synthase mutants even directly synthesized SA from chorismate (Plach et

562 al. 2015). We, thus, had a closer look at the putative chorismate binding enzymes of $T$.

563 azollae. Similar to our analyses for the anthranilate synthase of the host, we aligned the

564 protein sequences of $T$. azollae with that of the anthranilate synthase from $S$. enterica

565 subsp. enterica serovar Typhimurium str. LT2. As described for several major phyla of

566 bacteria (Plach et al., 2015), the anthranilate synthases of cyanobacteria had the QML motif,

567 and the cyanobacterial aminodeoxychorismate synthases encoded the expected QMI motif

568 (Figure 8d). Likewise, salicylate synthase and ICS encoded usual motifs with KLS and KLV,

569 respectively (Figure 8d).

570

571 Cyanobacterial anthranilate synthase, including that of $T$. azollae, and

572 aminodeoxychorismate synthase have the QML and QMI motif. Hence, they show the typical

573 aa pattern for these types of enzymes (Plach et al. 2015) and are likely not involved in SA

574 biosynthesis. The ICS had a KLV motif, frequently found for bacterial ICS sequences (Plach

575 et al. 2015). This suggests that $T$. azollae is capable of synthesizing isochorismate but not

576 SA. Given that Trichormus species have been shown to produce SA (Toribio et al. 2020), it

577 can be envisioned that $T$. azollae lost its salicylate synthase or IPL. Indeed, during the 66-

578100 million years of co-evolution between fern and cyanobiont (Hall and Swanson 1968,

579 Collinson 2002; Carrapiço 2006), the genome has started to erode, evident by a high

580 proportion of pseudogenes (Ran et al. 2010). It could therefore, be that due to SA being

581 synthesized by Azolla, T. azollae lost its SA synthesizing capabilities. Isochorismate in

582 bacteria can be funneled into the menaquinone pathway (Daruwala et al. 1996, Meganathan

583 and Kwon 2009) or to produce SA under iron deficiency, possibly to act as a siderophore

584 (Visca et al. 1993). In the here described scenario isochorismate of the cyanobiont would

585 singularly be used for menaquinone biosynthesis, while potential SA-associated

586 siderophores may require SA from Azolla to be shuffled to its symbiont. However,

587 isochorismate can decompose at slow rates directly into SA and the existence of an IPL may

588 not be necessary when the other isochorismate-metabolizing route towards menaquinone is

589 not active (Rekhter et al. 2019). 
591 PAL-dependent routes appear the most likely path towards SA in Azolla

592 All major land plant lineages and green algae appear to encode an ICS sequence in their 593 genomes (Figure 1a). This includes the only other sequenced fern genome of Salvinia 594 cucullata-but not that of $A$. filiculoides. This warrants attention. If we rule out simple 595 technical errors, $A$. filiculoides appears to have secondarily lost its ICS sequence and by that 596 its ability to synthesize isochorismate. It is conceivable that during the evolutionary history of 597 plants and algae, SA biosynthesis had already once been recruited from a cyanobiont-the 598 cyanobacterial plastid progenitor (Gross et al., 2006). In the Brassicaceae $A$. thaliana ICS is 599 localized to the plastid, where it synthesizes isochorismate (Wildermuth et al. 2001, Garcion 600 et al. 2008), which is then transferred to the cytoplasm to be further processed into SA 601 (Rekhter et al. 2019). It is thus tempting to speculate that this is a case where the tape of 602 evolution is replayed: the fern Azolla receives isochorismate from its symbiont, T. azollae, 603 and uses this backbone molecule to synthesize SA. Indeed, the genome of $T$. azollae shows 604 clear traces of an obligate symbiont and-based on its genome structure and its mode of 605 inheritance-was speculated to be an organelle in the making (Ran et al. 2010). Yet no 606 cases of endosymbiotic gene transfer have been reported for the genome of $A$. filiculoides 607 (Li et al. 2018). Indeed, cyanobiont-free Azolla species can survive with ample nitrogen 608 supply (Brouwer et al., 2017). Thus, while it is a fascinating area of speculation, we would 609 suggest that Azolla does not have to rely on the cyanobionts for the synthesis of its defense 610 hormone.

612 Overall, it appears to be more likely that Azolla uses the PAL-dependent pathway. This does 613 not exclude that $T$. azollae requires host-derived SA. Indeed, that both partners rely on the 614 phytohormone to some degree is in agreement with our previous data, where exogenous 615 application of an SA-derivative triggered alterations in host and cyanobiont gene expression 616 patterns as well as cyanobiont abundance (de Vries et al. 2018). The obvious question 617 following this is, whether SA biosynthesis and SA response can be modulated by the 618 cyanobionts. To gain first insights, we next investigated the expression profile and 619 differential gene expression (DGE) of the PAL-dependent biosynthesis pathway and SA 620 responses of $A$. filiculoides upon changes in nitrogen availability and presence/absence of 621 the cyanobiont.

623 Alterations in the cyanobionts population influence the gene expression patterns for 624 benzoic acid-derived SA biosynthesis

625 Nitrogen availability has been shown to modulate expression of genes involved in the 626 synthesis of phenylpropanoid-derived flavonoids in A. filiculoides (Güngör et al. 2021). 627 Indeed, the absence of cyanobionts also change expression levels of CHS in A. filiculoides 
628 (Eily et al. 2019), overall linking the phenylpropanoid-derived compounds to the symbiosis.

629 SA, which is a conceivable phenylpropanoid-derived compound in A. filiculoides, is also a

630 regulator of flavonoids (Nugroho et al. 2002, Tounekti et al. 2013, Ni et al. 2018). In bacteria,

631 SA is likely used as a siderophore to sequester iron from iron-poor environments (Visca et

632 al. 1993). Furthermore, in 2018 we have shown that SA can influence the population size of

633 the cyanobionts and their nitrogen fixation-related gene expression (de Vries et al., 2018).

634 To explore these factors, we (a) investigated the gene expression profiles upon these two

635 factors and (b) calculated the DGE for A. filiculoides with resepct to the PAL-dependent 636 pathway.

638 To understand the global gene expression patterns of genes for SA synthesis and down639 stream pathways in A. filiculoides, we first analyzed gene expression levels in Transcript Per 640 Million (TPM, supplemental data S2A and S3A). In total, we investigated 19 high-confidence 641 candidate genes; 18 of these showed an average TPM above 1 in at least one condition. $A$. 642 filiculoides invested a pronounced transcript budget into the phenylpropanoid pathway: while 643 the cumulated expression of all genes amounted to an average (over all conditions) of $6442734.79 \pm 544.91$ TPM, the 11 homologs of genes from the canonical phenylpropanoid 645 pathway (7 PAL-, and 4 4CL-homologs) made up 2335.55 \pm 535.27 TPM—which was largely 646 due to the PAL homologs. Of the seven putative PAL-coding genes, one distinguished itself 647 (Azfi_s0123.g048412) by dwarfing all the other average gene expression levels with a TPM 648 of $1757.05 \pm 338.51$. At the same time, Azfi_s0123.g048412 had the most stable expression 649 level, never showing differential gene expression changes under the conditions analyzed 650 here. For comparison, the TPM of $4 \mathrm{CL}$ amounted to $24.44 \pm 11.58$.

65211 of 19 high-confidence candidate genes for the SA biosynthesis pathway in $A$. filiculoides 653 showed significant changes in their differential gene expression patterns due to supply of 654 fixed nitrogen and/or presence of the cyanobiont (Benjamini Hochberg adjusted FDR $\leq 0.05$; 655 Figure 9a based on the pathways shown in Figure 7, Supplemental data S2B); 8 of these 11 656 genes were up or down-regulated with a $\log _{2}$ (fold-change[FC]) $>1$ or $<-1$. The absence of 657 fixed nitrogen alone in presence of the cyanobiont (nYcY vs. nNcY) or the absence of the 658 cyanobiont in presence of fixed nitrogen ( $\mathrm{nYcY}$ vs. $\mathrm{nYcN}$ ) has only a small effect on the 659 expression of the PAL-dependent biosynthesis pathway: only 3 of 19 genes showed 660 differential gene expression in either of the comparisons. Most changes were observed 661 when comparing the presence of cyanobionts ( $\mathrm{nNcY}$ ) to the absence of cyanobionts ( $\mathrm{nNcN}$ ) 662 in the absence of fixed nitrogen (8/19 differentially expressed genes) and when comparing 663 presence of fixed nitrogen and cyanobiont ( $\mathrm{nYcY}$ ) with the absence of both $(\mathrm{nNcN} ; 9 / 19$ 664 differentially expressed genes). These two comparisons shared seven differentially 
665 expressed genes, of which two (belonging to gene families $P A L$ and $B A L D H$ ) were up and 666 five were down-regulated (belonging to gene families $P A L, 4 C L, B A L D H$ and $A A O 4$ ) in both 667 conditions.

669 The two initial steps in the core phenylpropanoid pathway, $P A L$ and $4 C L$, the initial step in 670 the $\beta$-oxidative pathway (BZO1) and the last steps in the conversion of cytoplasmic 671 benzaldehyde to benzoic acid ( $A A O 4$ and $B A L D H)$ were responsive on a gene regulatory 672 level (Figure 9a). The peroxisomal steps catalyzed by gene families AIM1, DHNAT1/2 and $673 K A T$ showed little differential gene expression; only the AfKAT candidate was minimally 674 induced $\left(\log _{2}(F C)=0.38\right.$; FDR=0.02) when both nitrogen and cyanobiont conditions were 675 changed ( $\mathrm{nYcN}$ vs. $\mathrm{nNcY}$ ). This is in agreement with the phenylpropanoid pathway being 676 regulated on a transcriptional level (e.g. Weisshaar and Jenkins 1998, Deluc et al. 2006, Xie 677 et al. 2008). PAL genes are responsive to biotic stressors (Oliva et al. 2015, Reboledo et al. 678 2021). It is therefore, in general, not surprising that genes from the phenylpropanoid 679 pathway are responsive to the treatment. In addition, the late steps in the pathway are 680 modulated due to the availability of the cyanobiont and fixed nitrogen. Hence not only SA 681 appears to alter the abundance of the cyanobiont and expression of genes relevant for 682 nitrogen fixation (de Vries et al. 2018), availability of both components also influences SA 683 biosynthesis. This suggests an intricate system of communication between the two partners.

685 The pattern of how SA biosynthesis is regulated on the gene level appears complex. 686 Sometimes it is contrasting within a gene family or opposite responses occur when changes 687 in cyanobiont presence/absence is evaluated compared to when changes in the availability 688 of fixed nitrogen are evaluated. It appears that in the presence of cyanobionts more genes 689 are down-regulated than up-regulated, though (Figure 9a). Assuming that down-regulation of 690 genes in the SA biosynthesis pathway translates into less SA being produced, this appears 691 to be in contrast with SA levels being induced during colonization of some symbionts in other 692 plants (Fernández et al. 2014). However, it was noted that such defenses are early on 693 responses, hypothesized to stop symbionts from too much proliferation (Plett and Martin 694 2018). An integrated symbiont, however, is a long-term association. Thus, using SA to limit 695 proliferation within a host early on during symbiosis is not relevant. Moreover, in our 696 previous data on exogenous application of MeSA, we observed an increase of the 697 cyanobiont after treatment (de Vries et al. 2018). Taken together with the expression data on 698 the putative SA biosynthesis genes one may hypothesize a feedback loop between the 699 accumulation of SA and the accumulation of the cyanobiont. This is in agreement with MeSA 700 reducing the expression of the gene encoding iron-dependent niFE (de Vries et al. 2018). 
702 Expression profile of SA responsive pathways in $A$. filiculoides show distinct 703 responses towards the availability of fixed nitrogen and the cyanobiont

704 In several land plant species, SA levels are induced during interactions with beneficial 705 microbes (Blilou et al. 1999, Blilou et al. 2000, Liu et al. 2003, Pozo et al. 2015). Further

706 cyanobacterial treatment of $A$. thaliana resulted in the induction of SA controlled gene 707 expression (Belton et al. 2020). Above, we showed that gene expression levels associated 708 with SA biosynthesis are reduced in presence of the cyanobionts. Hence, we wondered 709 whether SA responsive genes may also differ in their gene expression in this particular 710 symbiosis compared to less intimate ones.

711

712 We downloaded all $A$. thaliana protein sequences of genes that are associated with the term 713 'SA responses' from TAIR (Lamesch et al., 2011). These 176 sequences were used as a 714 query in a reciprocal BLASTp approach, first against all proteins of $A$. filiculoides and the 715 thusly obtained homologs back against $A$. thaliana. In total we found 105 homologs, of which 71697 are high confidence proteins. These 97 homologs correspond to 47 different SA717 responsive proteins of $A$. thaliana involved in various processes (Figure 9b, Supplemental 718 data S3B). Overall, $55.7 \%$ of the homologs of SA-responsive genes show differential gene 719 expression $(F D R<0.05)$ in at least one comparison. Similar to the SA biosynthesis 720 expression, SA-derived responses showed most differential expressed genes in the 721 comparisons nNcY vs. $n N c N$ (31/97 homologs) and $n Y c Y$ vs. $n N c N$ (35/97 homologs). Of 722 those genes, 23 were shared between the conditions, nine were up-regulated and 14 down723 regulated $(F D R<0.05)$. Corroborating the results of the expression patterns of the SA 724 biosynthesis, changes in the availability of fixed nitrogen have only little influence on the 725 expression of SA responsive genes. Independent of the presence of the cyanobiont only two 726 ( $\mathrm{nYcY}$ vs. $\mathrm{nNcY}$ ) and seven genes ( $\mathrm{nYcN}$ vs. $\mathrm{nNcN}$ ) were significantly changed in their 727 expression (FDR <0.05). Of those only one (Azfi_s0427.g069291) showed the same 728 direction of regulation, and was induced in both treatments ( $\log _{2}$-foldchange $=1.64$ in $n Y c Y$ 729 vs. $\mathrm{nNcY}$ and $\log _{2}$-foldchange=2.31 in $\mathrm{nYcN}$ vs. $\mathrm{nNcN}$ ). Azfi_s0427.g069291 was also 730 induced in comparisons where presence of fixed nitrogen and cyanobiont was changed 731 (FDR<0.05). Azfi_s0427.g069291 encodes a putative 2-oxoglutarate (2OG)/Fe(II)732 dependent oxygenase (2-ODD) superfamily protein. Given the diversity of reactions in 733 specialized metabolism and hormone homeodynamics that are catalyzed by 2-ODDs it is 734 difficult to make an educated prediction of what the regulation of this 2-ODD means. That said, it is an interesting candidate enzyme for future studies.

737 Overall, presence of nitrogen compared to its absence and presence of the cyanobiont 738 compared to its absence result in more genes associated with a response to SA being 
739 down-regulated than up-regulated (Figure 9b). This indicates that SA signaling is reduced in

740 the presence of fixed nitrogen. The observation was more pronounced when the presence of

741 the cyanobiont was altered than when the availability of fixed nitrogen was changed. This is

742 due to the generally low transcriptional response to changes in fixed nitrogen. In contrast to

743 the abundance of down-regulated genes in response to a change of one component in the

744 system, changes in both components lead to a complex dysregulation of putative SA-

745 responsive genes (Figure 9b).

746

747 Many of the differentially expressed genes are found in the category of transcription factors

748 and MAP kinases. Here most homologs appear down-regulated when the presence of the

749 cyanobiont is compared to its absence, independent of the availability of nitrogen (FDR

$750<0.05$, Figure 9b). In the comparison of $n Y c Y$ vs. $n Y c N$ eight TFs are down-regulated and

751 only three are up-regulated. Also, two MAP kinase homologs are down-regulated and none

752 are up-regulated. In $\mathrm{nNcY}$ vs. $\mathrm{nNcN} 10$ TFs and four MAP kinases show down-regulation,

753 while five TFs and no MAP kinase show up-regulation. It appears that especially up-stream

754 regulators of defense responses are affected by the presence of the cyanobiont. This is in

755 agreement with the reduction of gene expression of the putative SA biosynthesis pathway

756 we observed in the same comparisons (Figure 9a). In contrast, SA signaling was induced

757 upon treatment of $A$. thaliana with Nostoc punctiforme PCC 73102 (Belton et al. 2020), a

758 cyanobacterium from the same family as Azolla's cyanobiont. N. punctiforme PCC 73102 is

759 a strain with symbiotic competence that is often used in experiments with cyanobacteria and

760 different hosts (Meeks et al. 2001, Warshan et al. 2017). This fits the hypothesis of Plett and

761 Martin (2018), that early increase in SA during symbioses may be to establish the borders of

762 same associations. However, the association between T. azollae and the water fern is

763 perpetual. It is thus to be expected that the usual early responses to encountering potential

764 symbionts are not mirrored in this vertically inherited association. Besides, data on host-

765 symbiont interaction is often gathered from angiosperm systems. Data related to SA

766 signaling in other plants during interaction with pathogenic microorganisms already indicate

767 differences in the defense network; for example, the antagonism between SA and another

768 phytohormone, jasmonic acid, appears to not exist in spruce during infection with a

769 necrotrophic pathogen (Arnerup et al. 2013). Additionally, it appears that the genes coding

770 for the Arabidopsis SA receptors AtNPR1, 3 and 4, which have contrasting roles in SA

771 signaling (Ding et al. 2018), arose from a lineage-specific radiation ( $\mathrm{Li}$ et al. 2020,

772 supplemental data therein). In $A$. filiculoides two possible candidates for NPR homologs,

773 likely derived from a lineage-specific duplication, are found ( $\mathrm{Li}$ et al. 2020, supplemental data

774 therein). Euphyllophytes thus likely had only a single ancestral NPR protein. Given these 
775 data, it is reasonable to assume that SA signaling is in A. filiculoides in general and in the

776 symbiosis of the fern only partially similar to that of model angiosperms.

\section{Conclusion}

779 The phytohormone SA is important for biotic interactions in plants and iron-sequestration in 780 bacteria. Previously, we showed that SA is a candidate molecule involved in the tight 781 coordination of the symbiosis between A. filiculoides and its cyanobionts (de Vries et al., 782 2018). Here, we investigated the possible routes by which SA may be synthesized in 783 A. filiculoides as well as how the cyanobiont and the availability of fixed nitrogen influence gene expression patterns that can be associated with SA biosynthesis and signaling. Our data pinpoint that the most likely biosynthetic route towards SA in the fern derives from benzoic acid, rather than isochorismate. Although the cyanobiont is genetically capable of synthesizing the latter, it lacks genes encoding IPL or salicylate synthase to convert isochorismate or chorismate to SA. Most genes relevant for SA biosynthesis in the water fern as well as homologs to genes associated with response to SA are down-regulated in $A$. filiculoides with a cyanobiont compared to a Trichormus-free culture. Most of the differential regulation appears to be on up-stream regulators such as TFs and MAP kinases. Taken our previous and new data together, SA might participate in a feedback loop between $A$. filiculoides and T. azollae.

\section{ACKNOWLEDGEMENTS}

796 JdV thanks the European Research Council for funding under the European Union's Horizon 7972020 research and innovation programme (Grant Agreement No. 852725; ERC-StG 798 "TerreStriAL"). IF gratefully acknowledges support by the Deutsche 799 Forschungsgemeinschaft (INST 186/822-1).

TABLE

802

803 Table 1. Quantification of salicylic acid (SA) in root and green sporophyte tissues of 804 Azolla filiculoides via HPLC-MS/MS.

\begin{tabular}{lrrr}
\hline tissue type & biological replicate & weight f.w. [g] & SA [nmol/g f.w.] \\
& & & \\
\hline whole root & I & 0.02 & 0.22 \\
& II & 0.01 & 0.17 \\
III & 0.025 & 0.14 \\
sporophyte & I & 0.105 & 0.1 \\
& II & 0.1 & 0.11 \\
& III & 0.11 & 0.07 \\
\hline
\end{tabular}


FIGURE LEGENDS

Figure 1. Evolution of chorismate binding enzymes in the green lineage. (a) ML-

810 phylogeny of chorismate binding enzymes from Chloroplastida with 100 bootstrap replicates.

811 Sequences from $A$. thaliana are highlighted in pink and sequences of $A$. filiculoides are 812 highlighted in blue. We recovered three clear clades, one for isochorismate synthases (ICS),

813 one for aminodeoxychorismate synthases and $p$-aminobenzoate synthases and one for 814 anthranilate synthases. Bootstrap support of 100 is indicated by black dots on the branches.

815 On the right we indicate TIGR domain annotations for the for different categories of 816 chorismate binding enzymes. Black dots indicate which domain was found; no dot indicates 817 that no TIGR domain annotation was present for this sequence. White dots only occur for 818 collapsed branches, to indicate for which of the collapsed sequences a domain annotation 819 was available (black dot) and for which not (white dot). (b) Conserved sequence logos for 820 the clades containing anthranilate synthase, aminodeoxychorismate/p-aminobenzoate 821 synthase and isochorismate synthase (ICS) shown in (a). Highlighted residues correspond 822 to amino acids that have been suggested to be functionally relevant for the product 823 specificity of these enzyme categories in Plach et al. (2015).

Figure 2. Phylogeny of PAL and HAL sequences from eukaryotes and prokaryotes.

826 ML-phylogeny of eukaryotic phenylammonia lyases (PAL) and HALs with 100 bootstrap 827 replicates; $\mathrm{LG}+\mathrm{G} 4$ was chosen as model for protein evolution according to Bayesian 828 Information Criterion. Sequences for PAL in plants have been identified using an HMM 829 approach. Sequences from fungal PALs and eukaryote HALs have been selected from de 830 Vries et al. (2017). Sequences from $A$. thaliana have been highlighted in pink, $A$. filiculoides 831 is highlighted in blue, sequences from $S$. bicolor are indicated in black and TAM1 from Oryza 832 sativa is highlighted in orange. Bootstrap support of 100 is indicated by black and colored 833 dots on the branches. The clades with fungal PAL and plant tRNA synthase sequences have 834 been collapsed. The substrate-specificity defining residues 123 and 443 of S. bicolor PTAL 835 (Sb04g026510) have been analyzed for all sequences in the alignment and are indicated on 836 the right next to the respective sequences. One exception is the collapsed branch of fungal 837 PALs. Due to the variance of residue 123 the sequence logo for the two residues is printed 838 on the branch itself.

840 Figure 3. Phylogeny of Hydratases in Chloroplastida. ML-phylogeny pointing the 841 evolutionary history of AIM1/CHD hydratases in chloroplastida using 100 bootstrap 842 replicates; $\mathrm{LG}+\mathrm{I}+\mathrm{G} 4$ was chosen as model for protein evolution according to Bayesian 
843 Information Criterion. The AIM1 sequence of $A$. thaliana is highlighted in purple, its ortholog

844 PhCHD from Petunia hybrida is highlighted in light pink and sequence from $A$. filiculoides is

845 highlighted in blue. Bootstrap levels $=100$ are indicated by black dots.

Figure 4. ML-phylogeny determining the evolutionary history of KAT sequences in chloroplastida. The phylogeny is based on 100 bootstrap replicates; $L G+I+G 4$ was chosen as model for protein evolution according to Bayesian Information Criterion. Sequences from $A$. thaliana are shown in purple, the PhKAT sequence from $P$. hybrida is shown in light pink and the sequence from $A$. filiculoides is highlighted in blue. Clades with brassicaceae and angiosperm KAT sequences have been highlighted in purple, Solanaceae KAT sequences are indicated by a pink box and fern KAT sequences are indicated by a blue box. Bootstrap levels of a 100 are indicated by dots along the branches.

855

Figure 5. Phylogeny of the DHNAT family. ML-phylogeny was computed with IQ-Tree version multicore version 1.5 .5 for Linux 64-bit with 100 bootstrap-replicates; JTT+I+G4 was chosen as model for protein evolution according to Bayesian Information Criterion. Bootstrap support of 100 is indicated by black and colored dots on the individual branches. DHNAT1 and 2 from $A$. thaliana are indicated in purple, the monophyletic DHNAT1 clade is highlighted in purple and the monophyletic DHNAT2 clade is highlighted in pink. DHNAT orthologs in ferns are highlighted in blue and the sequences from $A$. filiculoides is written in blue and bold.

Figure 6. Evolution of the AAO family. ML-phylogeny with 100 bootstrap replicates to recover the evolution of the $A A O$ family; $L G+F+I+G 4$ was chosen as model for protein evolution according to Bayesian Information Criterion. The blastp searches identified sequences either belonging to xanthin dehydrogenase $(\mathrm{XDH})$ and $\mathrm{AAO}$ clades. The AAO clade is highlighted in purple and the $\mathrm{XDH}$ clade is highlighted in brown. Sequences from $A$.

870 thaliana are indicated in pink and sequences from $A$. filiculoides are indicated in blue.

871 Bootstrap support of 100 is indicated by black dots.

872

873 Figure 7. Routes of SA biosynthesis in land plants. SA can be derived from 874 isochorismate or benzoic acid. The route from isochorismate requires ICS and a GH3 875 enzyme (Wildermuth et al., 2001, Garcion et al. 2008, Rekhter et al. 2019, Torrens-Spence 876 et al. 2019), whereas the benzoic acid-derived SA pathway is less direct requires several 877 steps that function also in other pathways. The reactions are indicated by arrows and the 878 responsible enzymes are written on top of these arrows. Grey enzyme names indicate steps 879 that have not been analyzed in this study because (i) data was available from previous 
880

881

882

883

884

885

886

887

888

889

890

891

892

893

894

895

896

897

898

899

900

901

902

903

904

905

906

907

908

909

910

911

912

913

914

915

916

studies or (ii) sequences for these enzymes are not available from functionally characterized enzymes because there is only indirect evidence. Boxes below the arrows indicate the number of high-confidence sequences available in the genome of $A$. filiculoides. Grey boxes indicate absence in $A$. filiculoides, blue boxes indicate the presence of homologs in the fern and pink boxes indicate presence of orthologs encoded in the genome of $A$. filiculoides.

Figure 8. Chorismate-binding enzymes in cyanobacteria. (a) Three chorismate-binding enzymes, an isochorismate (ICS), anthranilate synthase (AS) and aminodeoxychorismate synthase (ADCS), were identified in T. azollae using a blastp search. Protein domains identified via TIGR are drawn to scale (blue= isochorismate synthase domain, yellow = anthranilate synthase component I, red = ADCS component I domain). Amino acid positions are indicated by the bar above. (b) Number of blastp hits for IPL in cyanobacteria, using the IPL sequence of $P$. aeruginosa sorted according to taxonomy. Hits recovering sequences from the Nostocales are highlighted by a brown box. (c) Number of hits recovered through a blastp search for salicylate synthase with the sequence from $M$. tuberculosis as query sorted according to cyanobacterial taxonomy. Hits to Nostocales are highlighted by a brown box. (d) Sequence logos for the conserved regions in chorismate binding enzymes. Highlighted in blue and orange are residues relevant for substrate specificity (Plach et al. 2015).

\section{Figure 9. Differential gene expression of homologs of genes for SA biosynthesis and}

SA responses. (a) Differential gene expression of genes associated with the PALdependent pathway for SA biosynthesis in A. filiculoides, including candidates for $A f P A L$, Af4CL, AfAAE clade VI (i.e. the closest homologs to AtBOZ1 and PhCNL in A. filiculoides), AfAIM1, AfKAT, AfDHNAT, AfBALDH, and AfAAO4. (b) Differential gene expression of homologs of SA-responsive genes. Significant differential regulation is indicated by blue and yellow colors. Light yellow indicates significant differential up-regulation (FDR $\leq 0.05$ ) with a $\log _{2}(\mathrm{FC})$ above 0 and below 1 , dark yellow indicates significant differential up-regulation $(F D R \leq 0.05)$ with a $\log _{2}(F C)$ above 1 . Light blue indicates differential significant downregulation (FDR $\leq 0.05)$ with a $\log _{2}(F C)$ below 0 but above -1 and dark blue indicates significant down-regulation $(F D R \leq 0.05)$ with a $\log _{2}(F C)$ below -1 .

\section{REFERENCES}

Adebesin F., Widhalm J.R., Lynch J.H., McCoy R.M. \& Dudareva N. (2018) A peroxisomal thioesterase plays auxiliary roles in plant $\beta$-oxidative benzoic acid metabolism. The Plant Journal 93, 905-916.

Amborella Genome Project (2013) The Amborella genome and the evolution of flowering plants. Science, 342, 1241089 
917 Ankenbauer R.G. \& Cox C.D. (1988) Isolation and characterization of Pseudomonas 918 aeruginosa mutants requiring salicylic acid for pyochelin biosynthesis. Journal of 919 Bacteriology 170, 5364-5367.

920 Argout, X., Salse, J., Aury, J.-M., Guiltinan, M. J., Droc, G., Gouzy, J., ... Lanaud, C. (2011). 921 The genome of Theobroma cacao. Nature Genetics, 43, 101-108.

Arnerup J., Nemesio-Gorriz M., Lundén K., Asiegbu F.O., Stenlid J. \& Elfstrand M. (2012)

Banks, J. A., Nishiyama, T., Hasebe, M., Bowman, J.L., Gribskov, N., dePamhilis, C., Albert, V. A., ... Grigoriev, I. V. (2011). The Selaginella genome identifies genetic changes associated with the evolution of vascular plants. Science, 332, 960-963.

Becking J.H. (1987) Endophyte transmission and activity in the Anabaena-Azolla association. Plant and Soil 100, 183-212.

Belton S., McCabe P.F. \& Ng C.K.Y. (2021) The cyanobacterium, Nostoc punctiforme can protect against programmed cell death and induce defence genes in Arabidopsis thaliana. Journal of Plant Interactions 16, 64-74.

Blilou I., Ocampo J., \& García Garrido J. (1999) Resistance of pea roots to endomycorrhizal fungus or Rhizobium correlates with enhanced levels of endogenous salicylic acid. Journal of Experimental Botany 50, 1663-1668.

Blilou I., Ocampo J., \& Garcia-Garrido J. 2000. Induction of Ltp (lipid transfer protein) and $P A L$ (phenylalanine ammonia lyase) gene expression in rice roots colonized by the arbuscular mycorrhizal fungus Glomus mossae. Journal of Experimental Botany 51, 1969-1977.

Blum M., Chang H.-Y., Chuguransky S., Grego T., Kandasaamy S., Mitchell A., ... Finn R.D. (2021) The InterPro protein families and domains database: 20 years on. Nucleic Acids Research 49, D344-D354.

Block A., Widhalm J.R., Fatihi A., Cahoon R.E., Wamboldt Y., Elowsky C., ... Basset G.J. (2014) The Origin and Biosynthesis of the Benzenoid Moiety of Ubiquinone (Coenzyme Q) in Arabidopsis. The Plant Cell 26, 1938-1948.

Bowman, J. L., Kohchi, T., Yamato, K.T., Jenkins, J., Shu, S., Ishizaki, K., ... Schmutz, J. (2017). Insights into land plant evolution garnered from the Marchantia polymorpha genome. Cell, 171, 287-304.

951 Schluepmann H. (2017) Metabolic Adaptation, a Specialized Leaf Organ Structure and

952 Vascular Responses to Diurnal N2 Fixation by Nostoc azollae Sustain the Astonishing 
953

954

955

956

957

958

959

960

961

962

963

964

965

966

967

968

969

970

971

972

973

974

975

976

977

978

979

980

981

982

983

984

985

986

987

988

989

Productivity of Azolla Ferns without Nitrogen Fertilizer. Frontiers in Plant Science 8, 1516.

Bussell J.D., Reichelt M., Wiszniewski A.A.G., Gershenzon J. \& Smith S.M. (2014) Peroxisomal ATP-Binding Cassette Transporter COMATOSE and the Multifunctional Protein ABNORMAL INFLORESCENCE MERISTEM Are Required for the Production of Benzoylated Metabolites in Arabidopsis Seeds. Plant Physiology 164, 48-54.

Calvert H.E. \& Peters G.A. (1981) The Azolla-Anabaena azollae relationship. New Phytologist 89, 327-335.

Calvert H.E., Pence M.K. \& Peters G.A. (1985) Ultrastructural ontogeny of leaf cavity trichomes in Azolla implies a functional role in metabolite exchange. Protoplasma 129, $10-27$.

Carpenter E.J., Matasci N., Ayyampalayam S., Wu S., Sun J., Yu J., ... Wong G.K.-S. (2019) Access to RNA-sequencing data from 1,173 plant species: The 1000 Plant transcriptomes initiative (1KP). GigaScience 8, giz126.

Carrapiço F (2006) Is the Azolla-Anabaena symbiosis a co-evolution case? In: Sitnykov A (ed) General botany: traditions and perspectives. Kazan University, Kazan, pp 193195

Cheng, S., Xian, W., Fu, Y., Marin, B., Keller, J., Wu, T., ... Melkonian, M. (2019) Genomes of subaerial Zygnematophyceae provide insights into land plant evolution. Cell, 179, 1057-1067.e14.

Cochrane F.C., Davin L.B. \& Lewis N.G. (2004) The Arabidopsis phenylalanine ammonia lyase gene family: kinetic characterization of the four PAL isoforms. Phytochemistry 65, 1557-1564.

Collinson M.E. (2002) The ecology of Cainozoic ferns. Review of Palaeobotany and Palynology 119, 51-68.

Colquhoun T.A., Marciniak D.M., Wedde A.E., Kim J.Y., Schwieterman M.L., Levin L.A., ... Clark D.G. (2012) A peroxisomally localized acyl-activating enzyme is required for volatile benzenoid formation in a Petuniaxhybrida cv. 'Mitchell Diploid' flower. Journal of Experimental Botany 63, 4821-4833.

Coquoz J.-L., Buchala A. \& Métraux J.-P. (1998) The Biosynthesis of Salicylic Acid in Potato Plants. Plant Physiology 117, 1095-1101.

Danielsson M., Lundén K., Elfstrand M., Hu J., Zhao T., Arnerup J., ... Stenlid J. (2011) Chemical and transcriptional responses of Norway spruce genotypes with different susceptibility to Heterobasidion spp. infection. BMC Plant Biology 11, 154.

Daruwala R., Kwon O., Meganathan R. \& Hudspeth M.E.S. (1996) A new isochorismate synthase specifically involved in menaquinone (vitamin $\mathrm{K}_{2}$ ) biosynthesis encoded by the menF gene. FEMS Microbiology Letters 140, 159-163. 
990 de Vries J., de Vries S., Slamovits C.H., Rose L.E. \& Archibald J.M. (2017) How Embryophytic is the Biosynthesis of Phenylpropanoids and their Derivatives in Streptophyte Algae? Plant and Cell Physiology 58, 934-945.

de Vries S., de Vries J., von Dahlen J.K., Gould S.B., Archibald J.M., Rose L.E. \& Slamovits C.H. (2018) On plant defense signaling networks and early land plant evolution. Communicative \& Integrative Biology 11, 1-14.

de Vries S., de Vries J., Teschke H., von Dahlen J.K., Rose L.E. \& Gould S.B. (2018) Jasmonic and salicylic acid response in the fern Azolla filiculoidesand its cyanobiont. Plant, Cell \& Environment 41, 2530-2548.

de Vries S. \& de Vries J. (2018) Azolla: A Model System for Symbiotic Nitrogen Fixation and Evolutionary Developmental Biology. In Current Advances in Fern Research. pp. 2146. Springer International Publishing, Cham.

de Vries J., Curtis B.A., Gould S.B. \& Archibald J.M. (2018) Embryophyte stress signaling evolved in the algal progenitors of land plants. Proceedings of the National Academy of Sciences of the United States of America 115, E3471-E3480.

de Vries J., Vries S., Curtis B.A., Zhou H., Penny S., Feussner K., ... Archibald J.M. (2020) Heat stress response in the closest algal relatives of land plants reveals conserved stress signaling circuits. The Plant Journal 324, 1064-24.

Deluc L., Barrieu F., Marchive C., Lauvergeat V., Decendit A., Richard T., ... Hamdi S. (2006) Characterization of a grapevine R2R3-MYB transcription factor that regulates the phenylpropanoid pathway. Plant Physiology 140, 499-511.

Ding Y., Sun T., Ao K., Peng Y., Zhang Y., Li X. \& Zhang Y. (2018) Opposite roles of salicylic acid receptors NPR1 and NPR3/NPR4 in transcriptional regulation of plant immunity. Cell 173, 1454-1467.e15.

Ding P. \& Ding Y. (2020) Stories of Salicylic Acid: A Plant Defense Hormone. Trends in Plant Science 25, 549-565.

Dijkhuizen L.W., Brouwer P., Bolhuis H., Reichart G.-J., Koppers N., Huettel B., ... Schluepmann H. (2017) Is there foul play in the leaf pocket? The metagenome of floating fern Azollareveals endophytes that do not fix $\mathrm{N}$ 2but may denitrify. New Phytologist 217, 453-466.

Eily A.N., Pryer K.M. \& Li F.-W. (2019) A first glimpse at genes important to the AzollaNostoc symbiosis. Symbiosis 78, 149-162.

El-Gebali S., Mistry J., Bateman A., Eddy S.R., Luciani A., Potter S.C., Qureshi M., Richardson L.J., Salazar G.A., Smart A., Sonnhammer E.L.L., Hirsh L., Paladin L., Piovesan D., Tosatto S.C.E., \& Finn R.D. (2019) The Pfam protein families database in 2019. Nucleic Acids Research 47, D427-D432. 
1026 Fernández I., Merlos M., López-Ráez J.A., Martínez-Medina A., Ferrol N., Azcón C., ... Pozo

1027

1028

1029

1030

1031

1032

1033

1034

1035

1036

1037

1038

1039

1040

1041

1042

1043

1044

1045

1046

1047

1048

1049

1050

1051

1052

1053

1054

1055

1056

1057

1058

1059

1060

1061

M.J. (2014) Defense Related Phytohormones Regulation in Arbuscular Mycorrhizal Symbioses Depends on the Partner Genotypes. Journal of Chemical Ecology 40, 791803.

Fürst-Jansen J.M.R., de Vries S. \& de Vries J. (2020) Evo-physio: on stress responses and the earliest land plants. Journal of Experimental Botany 66, 4-16.

Gaille C., Kast P., \& Haas D. (2002) Salicylate biosynthesis in Pseudomonas aeroginosa. The Journal of Biological Chemistry 277, 21768-21775.

Garcion C., Lohmann A., Lamodière E., Catinot J., Buchala A., Doermann P. \& Métraux J.P. (2008) Characterization and Biological Function of the ISOCHORISMATE SYNTHASE2 Gene of Arabidopsis. Plant Physiology 147, 1279-1287.

Gross J., Cho W.K., Lezhneva L., Falk J., Krupinska K., Shinozaki K., ... Meurer J. (2006) A plant locus essential for phylloquinone (vitamin K1) biosynthesis originated from a fusion of four eubacterial genes. Journal of Biological Chemistry 281, 17189-17196.

Güngör E., Brouwer P., Dijkhuizen L.W., Shaffar D.C., Nierop K.G.J., Vos R.C.H., ... Schluepmann H. (2021) Azolla ferns testify: seed plants and ferns share a common ancestor for leucoanthocyanidin reductase enzymes. New Phytologist 229, 11181132.

Hall J.W., Swanson N.P. (1968) Studies on fossil Azolla: Azolla montana, a Cretaceous megaspore with many small floats. American Journal of Botany 55, 1055-1061.

Hill D.J. (1989) The control of the cell cycle in microbial symbionts. New Phytologist 112, $175-184$.

Hori, K., Maruyama, F., Fujisawa, T., Togashi, T., Yamamoto, N., Seo, M., ... Ohta, H. (2014). Klebsormidium flaccidum genome reveals primary factors for plant terrestrial adaptation. Nature Communications, 5, 3978.

Ibdah M., Chen Y.-T., Wilkerson C.G. \& Pichersky E. (2009) An Aldehyde Oxidase in Developing Seeds of Arabidopsis Converts Benzaldehyde to Benzoic Acid. Plant Physiology 150, 416-423.

Iven T., König S., Singh S., Braus-Stromeyer S.A., Bischoff M., Tietze L.F., Braus G.H., Lipka V., Feussner I., Dröge-Laser W. (2012) Transcriptional activation and production of tryptophan-derived secondary metabolites in Arabidopsis roots contributes to the defense against the fungal vascular pathogen Verticillium longisporum. Molecular Plant 5, 1389-1402.

Jiao, C., Sørensen, I., Sun, X., Sun, H., Behar, H., Alseekh, S., ... Rose, J. K. C. (2020). The Penium margaritaceum genome: hallmarks of the origins of land plants. Cell, 181, P1097-1111.E12 
1062 Jones D.T., Taylor W.R. \& Thornton J.M. (1992) The rapid generation of mutation data matrices from protein sequences. Bioinformatics 8, 275-282.

1064

Jun S.-Y., Sattler S.A., Cortez G.S., Vermerris W., Sattler S.E. \& Kang C. (2018) Biochemical and Structural Analysis of Substrate Specificity of a Phenylalanine Ammonia-Lyase. Plant Physiology 176, 1452-1468.

Kalyaanamoorthy S., Minh B.Q., Wong T.K.F., von Haeseler A. \& Jermiin L.S. (2017) ModelFinder: fast model selection for accurate phylogenetic estimates. Nature methods 14, 587-589.

1070

Katoh K. \& Standley D.M. (2013) MAFFT Multiple Sequence Alignment Software Version 7:

1071

1072

1073

1074

1075

1076

1077

1078

1079

1080

1081

1082

1083

1084

1085

1086

1087

1088

1089

1090

1091

1092

1093

1094

1095

1096

1097 Improvements in Performance and Usability. Molecular Biology and Evolution 30, 772780.

Klempien A., Kaminaga Y., Qualley A., Nagegowda D.A., Widhalm J.R., Orlova I., ... Dudareva N. (2012) Contribution of CoA Ligases to Benzenoid Biosynthesis in Petunia Flowers. The Plant Cell 24, 2015-2030.

Kolappan S., Zwahlen J., Zhou R., Truglio J.J., Tonge P.J. \& Kisker C. (2007) Lysine 190 Is the Catalytic Base in MenF, the Menaquinone-Specific Isochorismate Synthase from Escherichia coli $\square$ : Implications for an Enzyme Family ${ }^{\dagger}$. Biochemistry 46, 946-953.

Kwon O. \& Meganathan R. (2009) Biosynthesis of Menaquinone (Vitamin $\mathrm{K}_{2}$ ) and Ubiquinone (Coenzyme Q). EcoSal Plus 3.

Lamesch, P., Berardini, T. Z., Li, D., Swarbreck, D., Wilks, C., Sasidharan, R., ... Huala, E. (2011). The Arabidopsis Information Resource (TAIR): improved gene annotation and new tools. Nucleic Acids Research, 40, D1202-D1210.

Lang, D., Ullrich, K. K., Murat, F., Fuchs, J., Jenkins, J., Haas, F. B., ... Rensing, S. A. (2018) The Physcomitrella patens chromosome-scale assembly reveals moss genome structure and evolution. The Plant Journal, 93, 515-533.

Le S.Q. \& Gascuel O. (2008) An improved general amino acid replacement matrix. Molecular Biology and Evolution 25, 1307-1320.

Lee S., Kaminaga Y., Cooper B., Pichersky E., Dudareva N. \& Chapple C. (2012) Benzoylation and sinapoylation of glucosinolate R-groups in Arabidopsis: Acylation of glucosinolates in Arabidopsis. The Plant Journal 72, 411-422.

Leon J., Shulaev V., Yalpani N., Lawton M.A. \& Raskin I. (1995) Benzoic acid 2-hydroxylase, a soluble oxygenase from tobacco, catalyzes salicylic acid biosynthesis. Proceedings of the National Academy of Sciences 92, 10413-10417.

Li F.-W., Brouwer P., Carretero-Paulet L., Cheng S., de Vries J., Delaux P.-M., .. Pryer K.M. (2018) Fern genomes elucidate land plant evolution and cyanobacterial symbioses. Nature Plants 4, 460-472. 
1098

1099

1100

1101

1102

1103

1104

1105

1106

1107

1108

1109

1110

1111

1112

1113

1114

1115

1116

1117

1118

1119

1120

1121

1122

1123

1124

1125

1126

1127

1128

1129

1130

1131

1132

Li F.-W., Nishiyama T., Waller M., Frangedakis E., Keller J., Li Z., ... Szövényi P. (2020) Anthoceros genomes illuminate the origin of land plants and the unique biology of hornworts. Nature Plants 6, 259-272.

Liu J., Blaylock L., Endre G., Cho J., Town C., VandenBosch K., \& Harrison M. (2003) Transcript profiling coupled with spatial expression analysis reveals genes involved in distinct developmental stages of the arbuscular mycorrhizal symbiosis. The Plant Cell 15, 2106-2123.

Long M.C., Nagegowda D.A., Kaminaga Y., Ho K.K., Kish C.M., Schnepp J., ... Dudareva N. (2009) Involvement of snapdragon benzaldehyde dehydrogenase in benzoic acid biosynthesis. The Plant Journal 59, 256-265.

Matyash V, Liebisch G, Kurzchalia TV, Shevchenko A, Schwudke D (2008) Lipid extraction by methyl-tert-butyl ether for high-throughput lipidomics. Journal of Lipid Research 49, 1137-1146.

Marchler-Bauer A. \& Bryant S.H. (2004) CD-Search: protein domain annotations on the fly. Nucleic Acids Research 32, W327-W331.

Meeks J.C., Elhai J., Thiel T., Potts M., Larimer F., Lamerdin J., Predki P., \& Atlas R. (2001) An overview of the genome of Nostoc punctiforme, a multicellular, symbiotic cyanobacterium. Photosynthesis Research 70, 85-106.

Meuwly P., Molders W., Buchala A. \& MCtraux J.-P. (1995) Local and systemic biosynthesis of salicylic acid in infected cucumber plants. Plant Physiology 109, 1107-1114.

Mistry J., Finn R.D., Eddy S.R., Bateman A. \& Punta M. (2013) Challenges in homology search: HMMER3 and convergent evolution of coiled-coil regions. Nucleic Acids Research 41, e121-e121.

Nguyen L.-T., Schmidt H.A., von Haeseler A. \& Minh B.Q. (2015) IQ-TREE: A Fast and Effective Stochastic Algorithm for Estimating Maximum-Likelihood Phylogenies. Molecular Biology and Evolution 32, 268-274.

Ni J., Dong L., Jiang Z., Yang X., Sun Z., Li J., ... Xu M. (2018) Salicylic acid-induced flavonoid accumulation in Ginkgo biloba leaves is dependent on red and far-red light. Industrial Crops and Products 118, 102-110.

Nishiyama, T., Sakayama, H., de Vries, J., Buschmann, H., Saint-Marcoux, D., Ullrich, K. K., ... Rensing, S. A. (2018). The Chara genome: secondary complexity and implications for plant terrestrialization. Cell, 174, 448-464.

Nugroho L.H., Verberne M.C. \& Verpoorte R. (2002) Activities of enzymes involved in the phenylpropanoid pathway in constitutively salicylic acid-producing tobacco plants. Plant Physiology and Biochemistry 40, 755-760. 
1133

1134

1135

1136

1137

1138

1139

1140

1141

1142

1143

1144

1145

1146

1147

1148

1149

1150

1151

1152

1153

1154

1155

1156

1157

1158

1159

1160

1161

1162

1163

1164

1165

1166

1167

1168

1169

Nystedt, B., Street, N. R., Wetterbom, A., Zuccolo, A., Lin, Y.-C., Scofield, D. G., ... Jansson, S. (2013). The Norway spruce genome sequence and conifer genome evolution. Nature, 497, 579-584.

Ouyang, S., Zhu, W., Hamilton, J., Lin, H., Campbell, M., Childs, K., ... Buell, C. R. (2007). The TIGR Rice Genome Annotation Resource: improvements and new features. Nucleic Acids Research, 35, D883-D887.

Pallas J.A., Paiva N.L., Lamb C. \& Dixon R.A. (1996) Tobacco plants epigenetically suppressed in phenylalanine ammonia-lyase expression do not develop systemic acquired resistance in response to infection by tobacco mosaic virus. The Plant Journal 10, 281-293.

Pereira A.L. \& Vasconcelos V. (2014). Classification and phylogeny of the cyanobiont Anabaena azollae Strasburger: An answered question? International Journal of Systematic and Evolutionary Microbiology, 64, 1830-1840.

Peters G.A., Toia R.E., Raveed D. \& Levine N.J. (1978) The Azolla-Anabaena azollae relationship. VI. Morphological aspects of the association. New Phytologist 80, 583593.

Peters G.A. \& Meeks J.C. (1989) The Azolla-Anabaena symbiosis: basic biology. Annual Review of Plant Biology 40, 193-210.

Pereira, A. L., \& Carrapiço, F. (2007). Histochemistry of simple hairs from the foliar cavities of Azolla filiculoides. Plant Biosystems, 141, 323-328.

Perkins S.K. \& Peters G.A. (1993) The Azolla-Anabaena symbiosis: endophyte continuity in the Azolla life-cycle is facilitated by epidermal trichomes. New Phytologist 123, 53-64.

Plach M.G., Löffler P., Merkl R. \& Sterner R. (2015) Conversion of Anthranilate Synthase into Isochorismate Synthase: Implications for the Evolution of Chorismate-Utilizing Enzymes. Angewandte Chemie International Edition 54, 11270-11274.

Plett J.M. \& Martin F.M. (2018) Know your enemy, embrace your friend: using omics to understand how plants respond differently to pathogenic and mutualistic microorganisms. The Plant Journal 93, 729-746.

Ponce De León I., Schmelz E.A., Gaggero C., Castro A., Álvarez A. \& Montesano M. (2012) Physcomitrella patens activates reinforcement of the cell wall, programmed cell death and accumulation of evolutionary conserved defence signals, such as salicylic acid and 12-oxo-phytodienoic acid, but not jasmonic acid, upon Botrytis cinerea infection: P. patens defence responses against B. cinerea. Molecular Plant Pathology 13, 960974.

Pozo M.J., López-Ráez J.A., Azcón-Aguilar C., \& García-Garrido J.M. (2015) Phytohormone as integrators of environmental signals in the regulation of mycorrhizal symbiosis. New Phytologist 205, 1431-1436. 
1170

1171

1172

1173

1174

1175

1176

1177

1178

1179

1180

1181

1182

1183

1184

1185

1186

1187

1188

1189

1190

1191

1192

1193

1194

1195

1196

1197

1198

1199

1200

1201

1202

1203

1204

Qualley A.V., Widhalm J.R., Adebesin F., Kish C.M. \& Dudareva N. (2012) Completion of the core -oxidative pathway of benzoic acid biosynthesis in plants. Proceedings of the National Academy of Sciences 109, 16383-16388.

Rai A.N., Soderback E. \& Bergman B. (2000) Cyanobacterium-plant symbioses. New Phytologist 147, 449-481.

Ran L., Larsson J., Vigil-Stenman T., Nylander J.A.A., Ininbergs K., Zheng W.-W., ... Bergman B. (2010) Genome Erosion in a Nitrogen-Fixing Vertically Transmitted Endosymbiotic Multicellular Cyanobacterium. PLoS ONE 5, e11486.

Reboledo G., d Agorio A., Vignale L., Batista-García R.A., Ponce De León I. (2021) Transriptional profiling reveals conserved and species-specific plant defense responses during the interaction of Physcomitrium patens with Botrytis cinerea. Plant Molecular Biology https://doi.org/10.1007/s11103-021-01116-0

Rekhter D., Lüdke D., Ding Y., Feussner K., Zienkiewicz K., Lipka V., ... Feussner I. (2019) Isochorismate-derived biosynthesis of the plant stress hormone salicylic acid. Science $365,498-502$.

Rippka R., Deruelles J., Waterbury J.B., Herdman M. \& Stanier R.Y. (1979) Generic assignments, strain histories and properties of pure cultures of cyanobacteria. Journal of General Microbiology 111, 1-61.

Robinson M.D. \& Oshlack A. (2010) A scaling normalization method for differential expression analysis of RNA-seq data. Genome Biology 11, R25.

Robinson M.D., McCarthy D.J., \& Smyth G.K. (2010) edgeR: A bioconductor package for differential expression analysis of digital gene expression data. Bioinformatics 26: $139-140$.

Shapiro S.S. \& Wilk M.B. (1965) An analysis of variance test for normality (complete samples). Biometrika, 591-611.

Shen H., Jin D., Shu J.-P., Zhou X.-L., Lei M., Wei R., ... Yan Y.-H. (2018) Large-scale phylogenomic analysis resolves a backbone phylogeny in ferns. GigaScience 7.

Shi D.-J. \& Hall D.O. (1988) TheAzolla-Anabaena association: Historical perspective, symbiosis and energy metabolism. The Botanical Review 54, 353-386.

Shockey J.M., Fulda M.S. \& Browse J. (2003) Arabidopsis contains a large superfamily of acyl-activating enzymes. Phylogenetic and biochemical analysis reveals a new class of acyl-coenzyme a synthetases. Plant Physiology 132, 1065-1076.

Sierro, N. Battey, J., Ouadi, S., Bakaher, N., Bovet, L., Willig, A., Goepfert, S., Peitsch, M. C., \& Ivanov, N. V. (2014). The tobacco genome sequence and its comparison with those of tomato and potato. Nature Communications, 5, 3833. 
1205 Slotte, T., Hazzouri, K. M., Ågren, J. A., Koenig, D., Maumus, F., Guo, Y.-L., ... Wrigth, S. I.

1206 (2013). The Capsella rubella genome and the genomic consequences of rapid mating

1207 system evolution. Nature Genetics, 45, 831-835

1208 Student (1908) The probable error of a mean. Biometrika 6, 1-25.

1209 The International Brachypodium Initiative. (2010). Genome sequencing and analysis of the 1210 model grass Brachypodium distachyon. Nature, 463, 763-768.

1211 Toribio A.J., Suárez-Estrella F., Jurado M.M., López M.J., López-González J.A. \& Moreno J.

1212 (2020) Prospection of cyanobacteria producing bioactive substances and their 1213 application as potential phytostimulating agents. Biotechnology Reports 26, e00449.

1214 Torrens-Spence M.P., Bobokalonova A., Carballo V., Glinkerman C.M., Pluskal T., Shen A. 1215 \& Weng J.-K. (2019) PBS3 and EPS1 Complete Salicylic Acid Biosynthesis from 1216 Isochorismate in Arabidopsis. Molecular Plant 12, 1577-1586.

Tounekti T., Hernández I. \& Munné-Bosch S. (2013) Salicylic Acid Biosynthesis and Role in Modulating Terpenoid and Flavonoid Metabolism in Plant Responses to Abiotic Stress. In Salicylic Acid. (eds S. Hayat, A. Ahmad \& M.N. Alyemeni), pp. 141-162. Springer Netherlands, Dordrecht.

Van Moerkercke A., Schauvinhold I., Pichersky E., Haring M.A. \& Schuurink R.C. (2009) A plant thiolase involved in benzoic acid biosynthesis and volatile benzenoid production. The Plant Journal 60, 292-302.

Visca P., Ciervo A., Sanfilippo V. \& Orsi N. (1993) Iron-regulated salicylate synthesis by Pseudomonas spp. Journal of General Microbiology 139, 1995-2001.

Vlot A.C., Dempsey D.M.A., \& Klessig D.F. (2009). Salicylic acid, a multifaceted hormone to combat disease. Annual Review of Phytopathology 47, 177-206.

Wan, T. et al. (2018). A genome for gnetophytes and early evolution of seed plants. Nature Plants, 4, 82-89.

Wang, S., Li, L., Li, H., Sahu, S. K., Wang, H., Xu, Y., ... Liu, X. (2020) Genomes of earlydiverging streptophyte algae shed light on plant terrestrialization. Nature Plants, 6, 95106.

Warshan D., Espinoza J.L., Stuart R.K., Richter R.A., Kim S.-Y., Shapiro N., .. Rasmussen U. (2017) Feathermoss and epiphytic Nostoc cooperate differently: expanding the spectrum of plant-cyanobacteria symbiosis. The ISME Journal 11, 2821-2833.

Weisshaar B. \& Jenkins G.I. (1998) Phenylpropanoid biosynthesis and its regulation. Current Opinion in Plant Biology 1, 251-257.

Wildermuth M.C., Dewdney J., Wu G. \& Ausubel F.M. (2001) Isochorismate synthase is required to synthesize salicylic acid for plant defence. Nature 414, 562-565.

1241

Widhalm J.R., Ducluzeau A.-L., Buller N.E., Elowsky C.G., Olsen L.J. \& Basset G.J.C. (2012) Phylloquinone (vitamin K1) biosynthesis in plants: two peroxisomal 
thioesterases of lactobacillales origin hydrolyze 1,4-dihydroxy-2-naphthoyl-coa: Plant DHNA-CoA thioesterases. The Plant Journal 71, 205-215.

1244 Widhalm J.R. \& Dudareva N. (2015) A Familiar Ring to It: Biosynthesis of Plant Benzoic Acids. Molecular Plant 8, 83-97.

1246 Yan J., Aboshi T., Teraishi M., Strickler S.R., Spindel J.E., Tung C.-W., ... Jander G. (2015) The Tyrosine Aminomutase TAM1 Is Required for $\beta$-Tyrosine Biosynthesis in Rice. The Plant Cell 27, 1265-1278.

Zheng W., Bergman B., Chen B., Zheng S., Xiang G. \& Rasmussen U. (2009a) Cellular responses in the cyanobacterial symbiont during its vertical transfer between plant generations in the Azolla microphyllasymbiosis. New Phytologist 181, 53-61.

Zheng W., Rang L., Bergman B. (2009b) Structural Characteristics of the CyanobacteriumAzolla Symbioses. In: Pawlowski K. (eds) Prokaryotic Symbionts in Plants. Microbiology Monographs, vol 8. Springer, Berlin, Heidelberg. of Mbtl, the Salicylate Synthase from Mycobacterium tuberculosis ${ }^{\dagger}$. Biochemistry 46, 
(b)
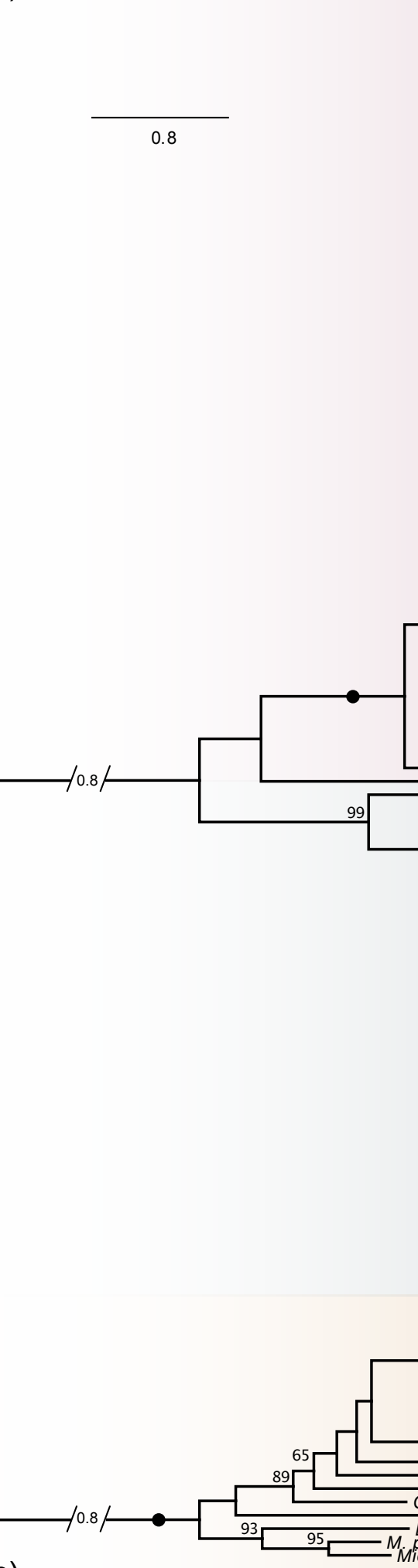

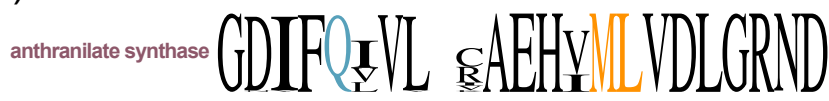

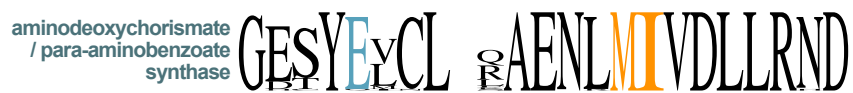

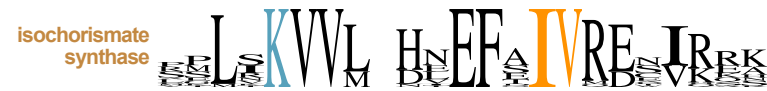


B. rapa Brara. G01580.1.p

B. oleracea Bol025102

B. rapa Brara. I03617.1.p

[98 C. grandiflora Cagra.0612s0051.1.p

A. thaliana AT3G53260 PAL2

98[ A. thaliana AT3G10340 PAL4

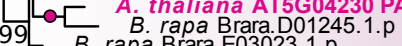

B. rapa Brara.E03023.1.p

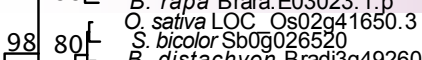

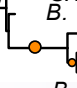

\section{S. bicolor Sb01g014020.p \\ O. sativa LOC Os11g48110.1 TAM1}

B. distachyon Bradi3g47120.1.p
B. distachyon Bradi3g47110.1.p

of distachyon Bradi3g49270.1.p

${ }_{93}$ B. distachyon Bradi3g48840.1.p

43 B. distachyon Bradi3g492801.

$76 \quad$ 93 || $\begin{aligned} & \text { O. sativa LOC Os05g35290.1 } \\ & \text { Sicolor Sb04g026540 PAL }\end{aligned}$

S. bicolor Sb04g026530 PAL

S. bicolor Sb04g026550 PAL

S. bicolor Sb04g026560 PAL

O. sativa LOC_Os02g41670.

O. sativa LOC-Os02g41680.1

O. sativa LOC Os04g43800.1

98 97- $B$. distachyon Bradi3g49250.2.p

85- 97 S. bicolor Sb04g026510 PTAL

O. sativa LOC_Os02g41630.2

68L S. bicolor Sb06g022740 PTAL

$P$. abies MA $10429279 \mathrm{~g} 0010$

A. filiculoides Azfi s0123.g048412

S. cucullata Sacu_v1.1s0091.g018764

A. filiculoides Azft s0096.g043679

62 - 4 S. cuculata Sacu v1.T s0253.g02679

77 G. montanum TnS000776579t01

of G. montanum TnS000998363t01

P. abies MA 44561g0010

어 $A$. agrestis BONN 228.5205.1

A. agrestis BONN 228.725.2
P.patens Pp3c14 11870V3.1.p

$P$. patens Pp3c1-18830V3.1.p
$P$ patens Pp3c1-18940V3 1.p

P.patens Pp3c1 18940V3.1.p

P. patens Pp3c24 13110V3.1.p

P. patens Pp3c21-7670V3.1.p

P. patens Pp3c21-7680V3.1.p

$P_{\dot{P}}$ patens Pp3c2 32410V3. 1.p

P. patens Pp3c2 30610V3.1.p

P. patens Pp3c10 21810V3.1.p

$P$ patens Pp3c19-13690V3.1

91 _ S cucullatā Sacu v1.1 s0067.g016271

A. filicullatä Sacu v1.1 s0067.g016271

A. filiculoides Azfi_s0205.g057722

A. filiculoides AzfI s0063.g035292

S. cucullata Sacu v1.1_s0017.g007129

S. cucullata Sacu v1.1 s001
P. abies MA 15852g0010
P. abies MA 73113g0010

S. cucullata Sacu v1.1 s001
P. abies MA 15852g0010
P. abies MA 73113g0010

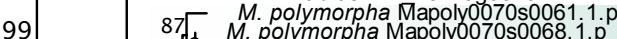

87 M. polymorpha Mapoly0070s0068.1.p

$62 \quad M$. polymorpha Mapoly0070s0071.1.p

M. polymorpha Mapoly0070s0065.1.p

73 - M. polymorpha Mapoly0009s0173.1.p

M. polymorpha Mapoly0044s0114.1.p

$M$. polymorpha Mapoly0132s0049.1.p

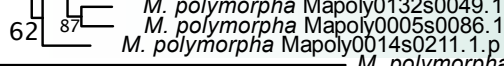

M. polymorpha Mapoly0142s0036.1.p

FK

\section{Monocot PALs}

TAM

\section{Monocot PAL}

PTAL

\section{Gymnosperm PAL}

\section{Fern PAL}

Gnetum PAL

Gymnosperm PAL

Gymnosperm PAL

Hornwort PAL

Moss PAL

Moss PAL

Fern PAL

Gymnosperm PAL

Gvmnosperm PAL

Liverwort PAL

Eukaryotic HAL

70 Blastocystis sp. subtype 4 XP 014528439.1 - Phytophthora sojae XP 009523491.1

Gallus gallus NP 989735.1

87 - Myotis brandtii XP 005882425.1

Eptesicus fuscus XP 008143014.1

99 [ Equus callabus XP 001494773.1

Equus callabus XP-005606583.

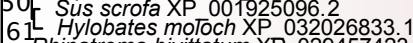

Rhinatrema bivittatum XP 029457423.1

Larimichthys crocea XP 010755594.

Hippoglossus hippoglossus XP 034444773.1

Daphnia magna XP 032788954.1

Daphnia magna XP-032788957. 


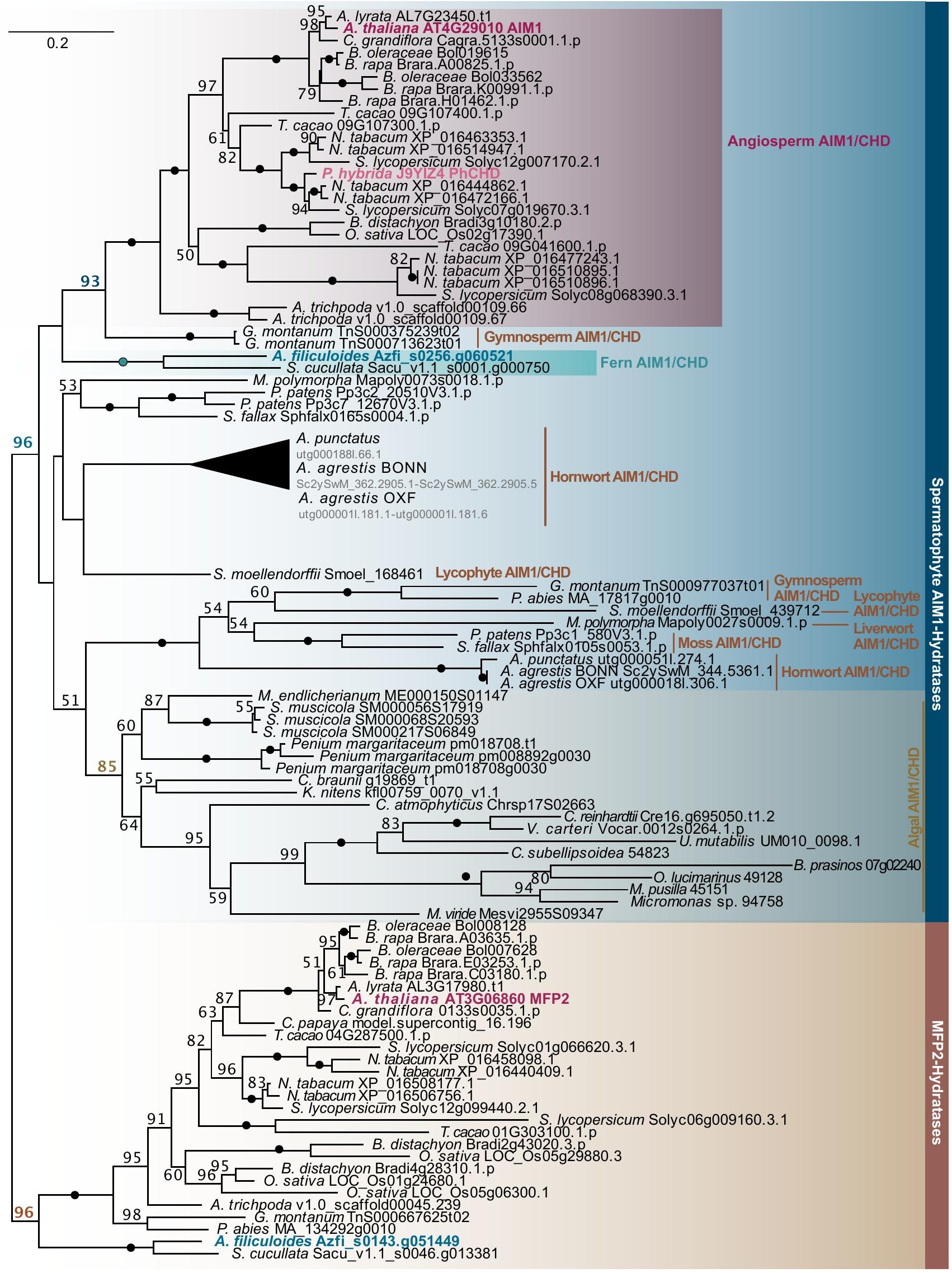


$74 \quad 78$ B. rapa Brara.E01079.1.p

66 B. Oleracea Boll13513

6 L B. rapa Brara.D02005.1.p

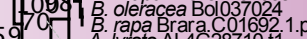

59 . A. lyrata AL 4G28710.t1.

80 6. Grandiflora Cagra.0301s0072.1.p

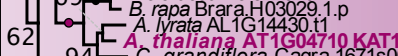

9 C. C. grandiflora Cagra.1671s0092.1.p

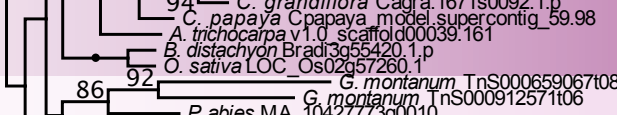

8692 -

S. moella montanum TnSo00729703t11

S. moendendorffii Smoel_447769

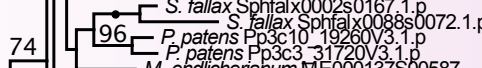
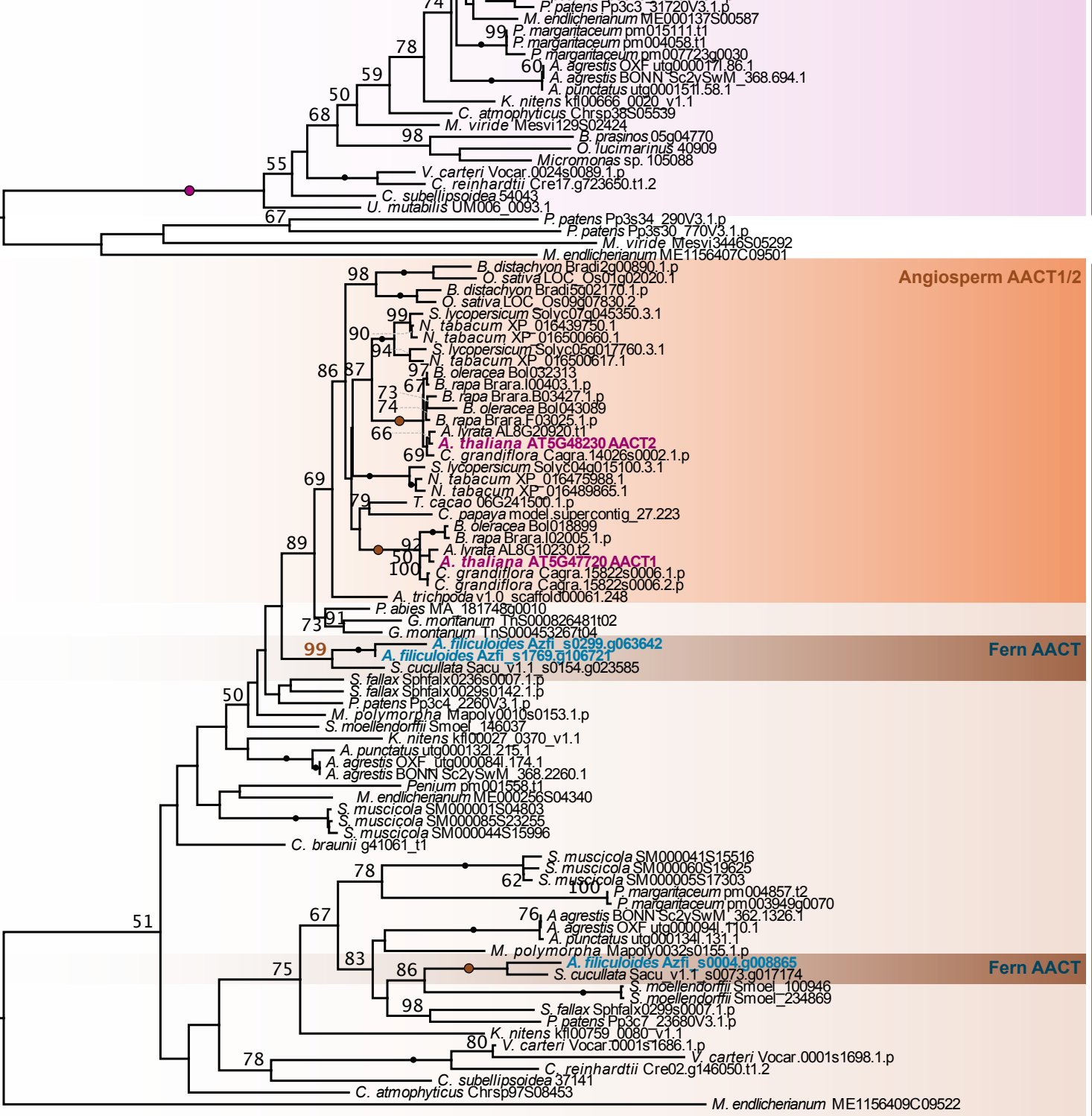


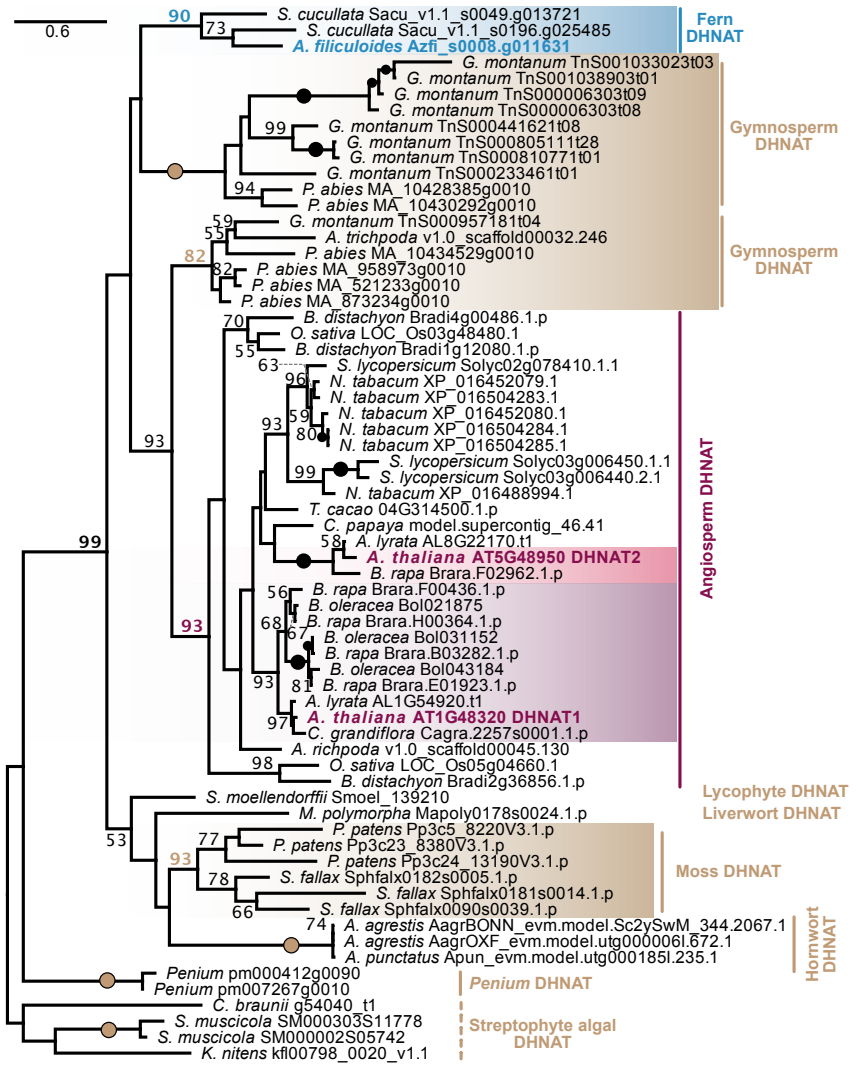




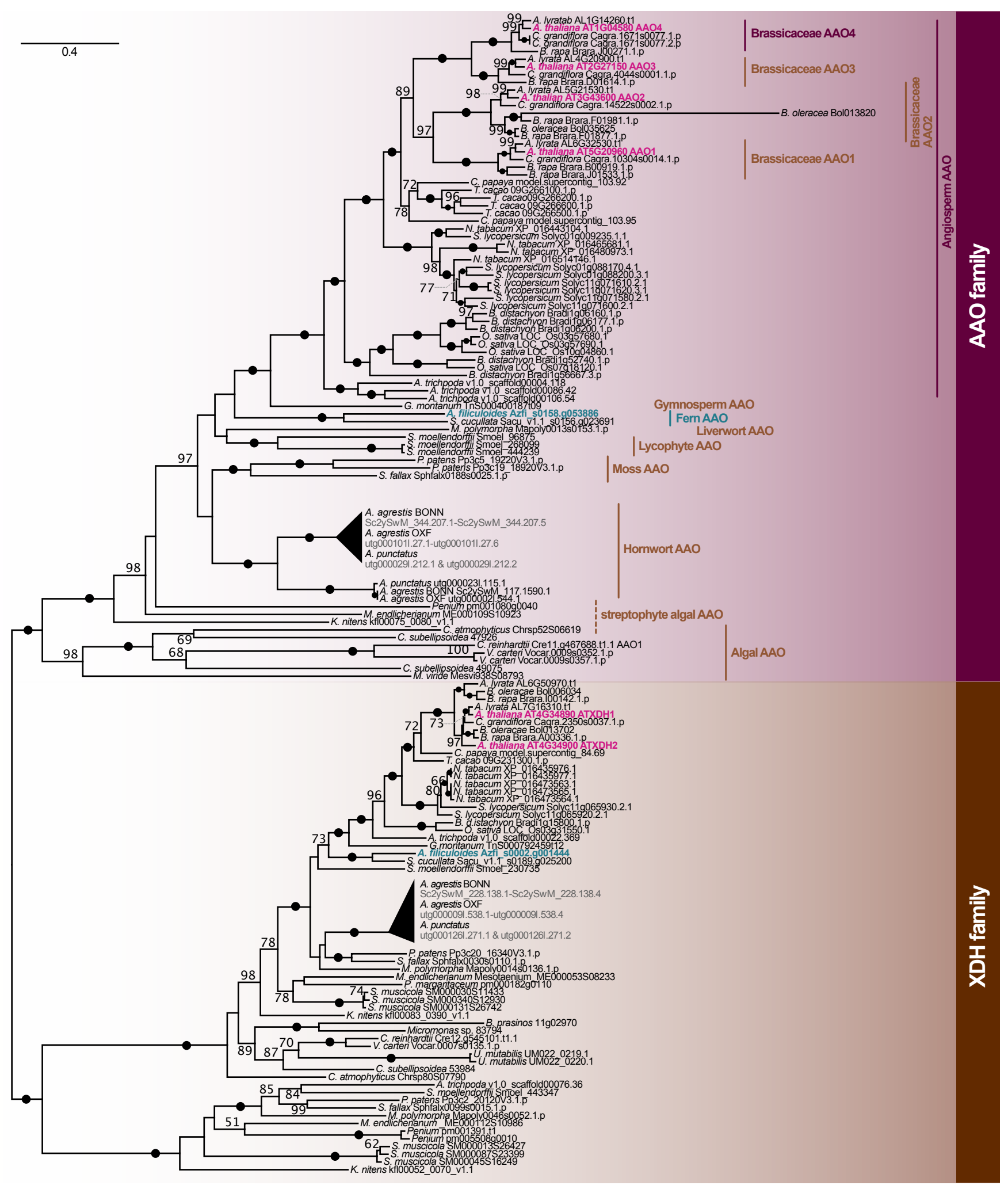




\section{CHLOROPLAST}

\section{CYTOSOL}

(l)

Chorismate

Isochorismate

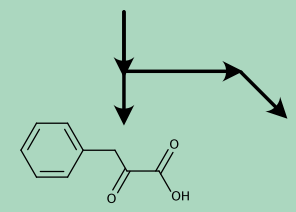

phenylpyruvate

?

$\downarrow$

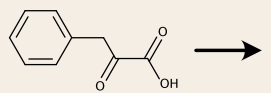

phenylpyruvate

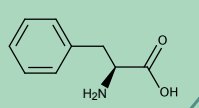

phenylalanine
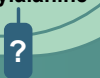<smiles>C=C(O[C@H]1C=CC=C(CC)[C@@H]1O)C(=O)O</smiles>

$\downarrow$
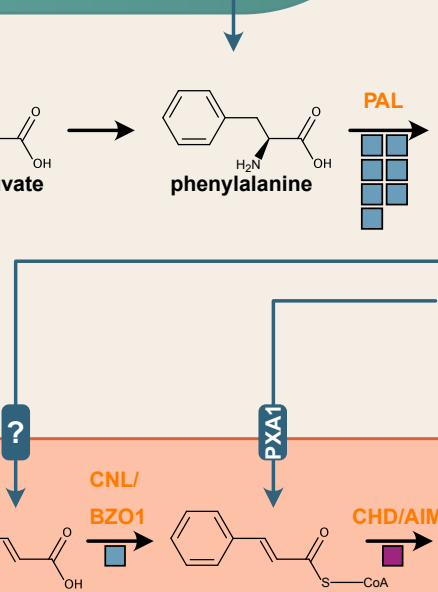

t-cinnamic acid

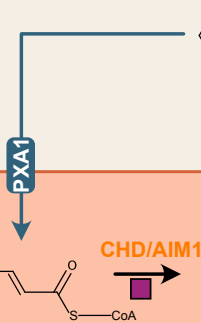

cinnamoyl-CoA

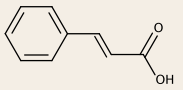

$t$-cinnamic acid

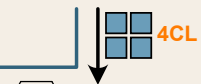

(1)

cinnamoyl-CoA

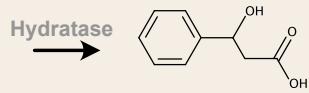

3-hydroxyphenylpropionic acid

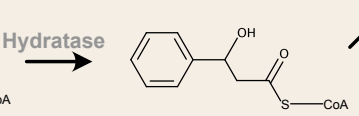

3-hydroxy-3-phenylproponoyl-CoA
MITOCHONDRION

CYP450 (BA2H)

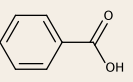

benzoic acid

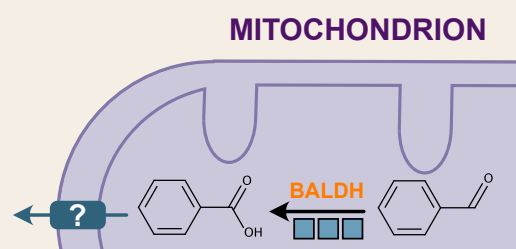

benzoic acid
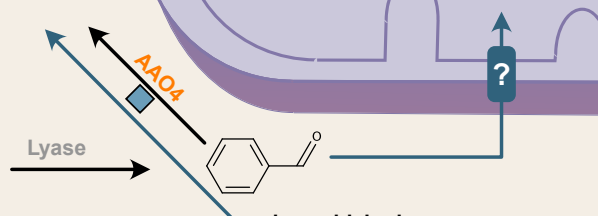

benzaldehyde
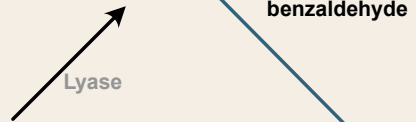

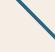
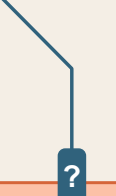

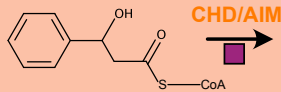

3-hydroxy-3-phenylproponoyl-CoA

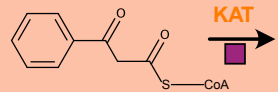

3-oxo-3-phenylproponoyl-CoA

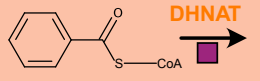

benzaldehyde

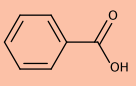

benzoic acid 


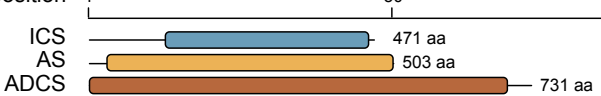

(b)

IPL

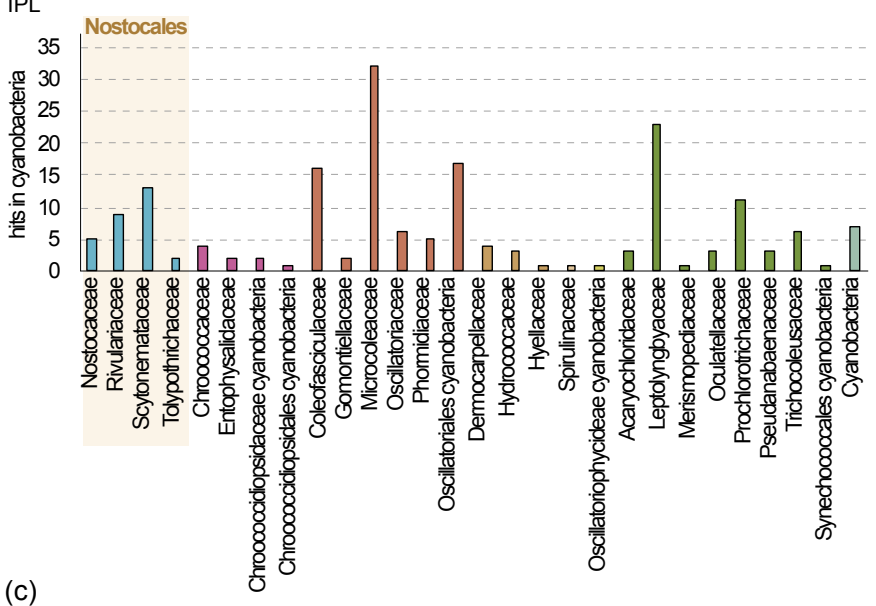

Salicylate synthase

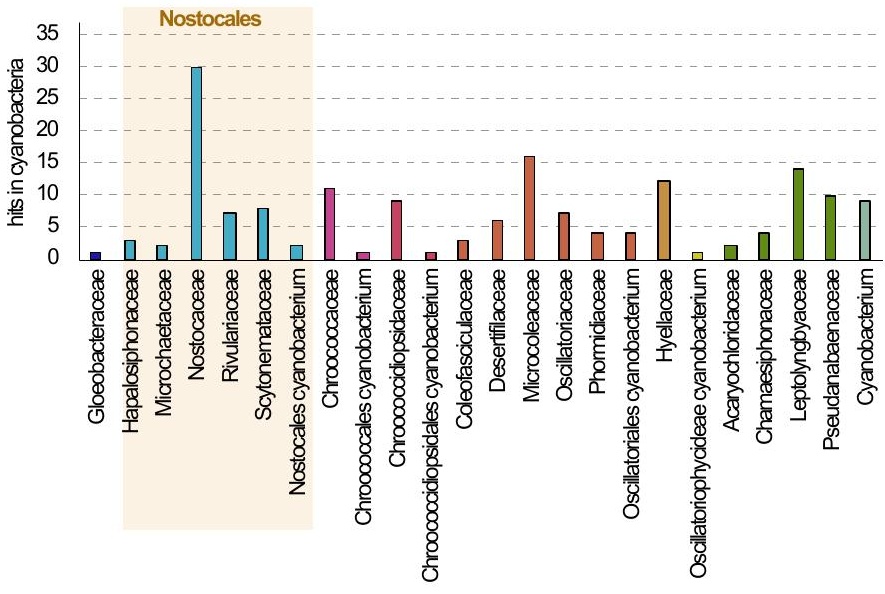

(d)

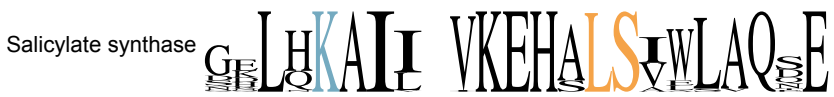
isochorismate synthase

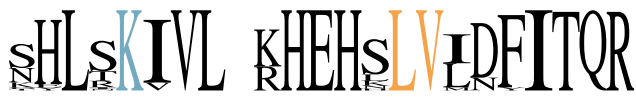

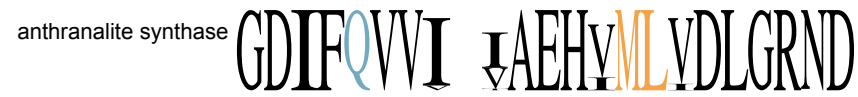
aminodeoxychorismate synthase

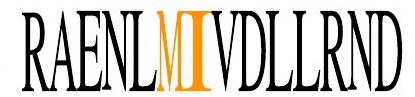


